\title{
Aeroelastic analyses of bridges using a Pseudo-3D vortex method and velocity-based synthetic turbulence generation
}

\author{
Igor Kavrakova,*, Guido Morgenthal $^{\mathrm{b}}$ \\ ${ }^{a}$ Research Training Group 1462, Bauhaus-University Weimar, Marienstr. 7, Weimar 99423, Germany \\ ${ }^{b}$ Chair of Modelling and Simulation of Structures, Bauhaus-University Weimar, Marienstr. 13, Weimar 99423, Germany
}

\begin{abstract}
The accurate representation of aerodynamic forces is essential for a safe, yet reasonable design of long-span bridges subjected to wind effects. In this paper, a novel extension of the Pseudo-three-dimensional Vortex Particle Method (Pseudo-3D VPM) is presented for Computational Fluid Dynamics (CFD) buffeting analysis of line-like structures. This extension entails an introduction of free-stream turbulent fluctuations, based on the velocity-based turbulence generation. The aerodynamic response of a longspan bridge is obtained by subjecting the 3D dynamic representation of the structure to correlated free-stream turbulence in twodimensional (2D) fluid planes, which are positioned along the bridge deck. The span-wise correlation of the free-stream turbulence between the 2D fluid planes is established based on Taylor's hypothesis of frozen turbulence. Moreover, the application of the laminar Pseudo-3D VPM is extended to a multimode flutter analysis. Finally, the structural response from the Pseudo-3D flutter and buffeting analyses is verified with the response, computed using the semi-analytical linear unsteady model in the time-domain. Meaningful merits of the turbulent Pseudo-3D VPM with respect to the linear unsteady model are the consideration of the 2D aerodynamic nonlinearity, nonlinear fluid memory, vortex shedding and local non-stationary turbulence effects in the aerodynamic forces. The good agreement of the responses for the two models in the 3D analyses demonstrates the applicability of the Pseudo-3D VPM for aeroelastic analyses of line-like structures under turbulent and laminar free-stream conditions.
\end{abstract}

Keywords: Computational Bridge Aerodynamics, Buffeting, Flutter, Long-span Bridges, Vortex Particle Method

\section{Introduction}

Wind-induced vibrations may constitute a governing criterion in the design of long-span bridges. Commonly, experimental and semi-analytical models have been used to compute the dynamic response of a bridge excited by gusty wind. In the recent past, numerical models based on Computational Fluid Dynamics (CFD) have gained substantial attention. The semianalytical models, aided by experimentally obtained aerodynamic coefficients, have been extensively used for aeroelastic analysis and are generally based on the quasi-steady or linear unsteady theory. Considering their underlying assumptions, the standard semi-analytical models tend to capture either the quasi-steady nonlinearity or linear fluid memory [1]. However, with increasing spans and sophisticated deck shapes, the linear and quasi-steady assumptions are challenged. Thus, the need to concurrently account for the nonlinearity and unsteadiness in the aerodynamic forces becomes apparent. Several semianalytical models have been developed to tackle this challenge [2-4]. Although very promising, none of the nonlinear models is able to fully describe the nonlinear and non-stationary aerodynamic behavior including all scales of turbulence and vortex shedding effects $[1,5,6]$. This explains why the utilization of

\footnotetext{
*Corresponding author. Tel. +49 (0) 3643584109

Email addresses: igor .kavrakov@uni-weimar.de (Igor Kavrakov), guido.morgenthal@uni-weimar.de (Guido Morgenthal)
}

reduced CFD models is appealing as an alternative in bridge aeroelasticity.

Complementary to the semi-analytical models, the application of CFD in bridge aerodynamics has gained momentum in the past few decades, offering substantial insight into the physics of fluid-structure interaction. Typically, the grid-based CFD methods such as the Finite Volume Method and the Finite Element Method have been used to determine the aerodynamic coefficients and seldom the aeroelastic response. As an alternative to the grid-based methods, the Lagrangian two-dimensional (2D) Vortex Particle Method (VPM) has proven to be a robust and efficient means for flow simulation in a relatively wide field of application, especially in free-field bluff body aerodynamics problems. In the VPM, the vorticity field is discretized by particles, carrying concentrated circulation. The VPM has been extensively utilized in bridge aerodynamics for the computation of the static wind coefficients and flutter derivatives [7-9], motion-induced indicial functions [10] and 2D vortex-induced vibrations [11].

Prendergast $[12,13]$ extended the 2D VPM to consider freestream turbulence by introducing the velocity-based turbulence generation (VTG). In this method, fluctuating random velocities are generated at the nodes of a ladder, positioned upstream of the section, as correlated multivariate stochastic processes. The velocities at the ladder nodes are then transformed into inflow particles, which are sequentially injected upstream into the domain. This simulates 2D turbulent field with prescribed spec- 
tral characteristics. The VTG has been successfully utilized for the determination of the aerodynamic admittance with analytical verification [14] and experimental validation [15]. Recently, the 2D VPM including VTG was compared in a synergistic manner with several semi-analytical models for aeroelastic analysis of bridges [16], yielding good correspondence in the linear range.

Generally, CFD has been rarely used for computation of a full-bridge three-dimensional (3D) response with laminar or turbulent free-stream. Since the present status of development on computational resources limits the possibilities for direct numerical simulation, reduced CFD models are warranted. To account for the 3D structural behavior while retaining the benefit of efficient of 2D CFD simulations, Morgenthal and McRobie [11] introduced the laminar Pseudo-3D VPM ${ }^{1}$ in bridge aerodynamics. In the Pseudo-3D VPM, the 3D structure is discretized using the beam elements, while the fluid domain is approximated by 2D planes (slices) positioned along the structural axis. This allows 3D representation of a line-like structure, considering an arbitrary number of modes, which are necessary for a proper representation of the dynamic behavior. The Pseudo-3D method was applied for the computation of vortex-induced vibrations of vertical steel masts [17] and the Niterói bridge [18], where the results were validated with experiments. An attempt was made in [19] to utilize the laminar Pseudo-3D method for to determine the critical flutter limit with few slices and as few as five modes. Nevertheless, the authors are not aware of any further applications application of the laminar Pseudo-3D VP$\mathrm{M}$ for high resolution multimode 3D flutter analysis including limit-cycle oscillations (LCO) of bridges.

Recently, Tolba and Mogenthal [20] extended the laminar Pseudo-3D VPM to account for the isotropic free-stream turbulence by introducing a particle-based turbulence generation (PTG). Unlike in the VTG, in this method, the circulation of the inflow particles is directly generated as a correlated random process, without prior generation of a velocity field. The correlation between slices is established in an empirical manner, imposing empirical coherence on the inflow particles directly during the generation process. The method was validated with experimental results to some extent; however, a comparison with a semi-analytical model was not reported. The motivation for the development of the PTG, instead of using the VTG for the Pseudo-3D buffeting analysis, was the inability of VTG method to retain the correlation between slices. The authors argued that with the VTG method "the correlations are not maintained in the process of their transformation to inflow vortex particles" [20] for the Pseudo-3D VPM. Nevertheless, it was not shown to what extent is the loss of the span-wise correlation.

In this brief overview, we have identified certain research gaps in the Pseudo-3D VPM framework from the aspect of methodology, application and comparison/verification with semianalytical models. Therefore, the goal of this study is to address these aspects to a certain extent. Specifically, we attempt to ex-

\footnotetext{
${ }^{1}$ By laminar Pseudo-3D VPM we denote a Pseudo-3D VPM with laminar free-stream, not a laminar flow. In the same manner we denote a turbulent Pseudo-3D VPM for turbulent free-stream.
}

tend the Pseudo-3D VPM by:

- introducing the VTG method for modeling of the freestream turbulence for the Pseudo-3D VPM and demonstrating its suitability by verifying the span-wise correlation;

- extending the applicability of the laminar Pseudo-3D VP$\mathrm{M}$ to multimode flutter analysis including LCO for longspan bridges;

- comparing the results from the flutter and buffeting analyses of the Pseudo-3D CFD model with the semi-analytical linear unsteady (LU) model in the time-domain.

The incentives to utilize the VTG instead of the PTG for the Pseudo-3D analysis are the meaningful advantages of: (i) partial consideration of anisotropy in the flow, and (ii) ability to prescribe the power spectral density (PSD) for both fluctuating components of the velocity. These two points, and the fact that a velocity field is generated instead of a vorticity makes the VT$\mathrm{G}$ method both more flexible and intuitive. Buffeting and flutter analyses, using the Pseudo-3D CFD and LU models, are performed for the Great Belt bridge and the results are compared. We attempt to interpret the results in such way, to illuminate, at least to a certain extent, some of the nonlinear phenomena such as LCO. The outcome of this study is intended to impact the civil and wind engineering communities from a scientific as well as from a practical aspect.

The article is organized as follows: The LU model is briefly outlined in Sec. 2. Section 3 presents the novel turbulent Pseudo3D VPM based on the VTG. Herein, we also briefly revisit the governing equations of the VPM, 2D VTG and laminar Pseudo3D VPM. A flow verification, buffeting and flutter analyses are performed for the Great Belt bridge in Sec. 4. Finally, concluding remarks are given in Sec. 5.

\section{Linear unsteady model}

In this section, we briefly outline the LU model, while detailed information can be found in the provided references. The equation of motion for a structural system in a dynamic equilibrium is defined in modal coordinates as follows:

$$
M \ddot{q}+C \dot{q}+K q=f
$$

where $\boldsymbol{q}=\boldsymbol{q}(t)$ is the modal displacement vector with its derivatives with respect to time $t, \dot{\boldsymbol{q}}=\dot{\boldsymbol{q}}(t)$ and $\ddot{\boldsymbol{q}}=\ddot{\boldsymbol{q}}(t)$, while $\boldsymbol{M}, \boldsymbol{C}$ and $\boldsymbol{K}$ represent the modal mass, modal damping and modal stiffness matrices, respectively. The modal force vector $\boldsymbol{f}=\boldsymbol{f}(t)$ is obtained from the mode-shape matrix $\boldsymbol{\Phi}$ and the external force vector $\boldsymbol{f}_{o}=\boldsymbol{f}_{o}(t)$ as $\boldsymbol{f}=\boldsymbol{\Phi}^{T} \boldsymbol{f}_{o}$.

The deck is discretized on beam finite elements, considering motion in the lateral $p=p(t)$, vertical $h=h(t)$ and rotational $\alpha=\alpha(t)$ direction. Correspondingly, the considered wind forces $f_{o} \in\{D, L, M\}$ acting on the deck are the drag $D=D(t)$, lift $L=L(t)$ and moment $M=M(t)$ forces. The standard coordinate system for applications in aeroelasticity is used, 
in which the lift and vertical displacements are positive downward, the drag and lateral displacements are positive downwind, while the rotation and pitching moment are positive in the clockwise direction (nose-up). The wind velocity is separated on a mean velocity $U$ and fluctuating components $u=u(t)$ and $w=w(t)$ in the lateral (positive downwind) and vertical direction (positive upward), respectively. In addition to the mean wind forces $\boldsymbol{f}_{s}$, Davenport [21] and Scanlan [22, 23] formulated the time-varying aerodynamic forces as a superposition of linear frequency-dependent functions due to structural motion (i.e. self-excited forces $f_{s e}$ ) and incoming turbulent wind (i.e. buffeting forces $\boldsymbol{f}_{b}$ ), that is, $\boldsymbol{f}_{o}=\boldsymbol{f}_{s}+\boldsymbol{f}_{s e}+\boldsymbol{f}_{b}$, by utilizing frequency-dependent coefficients. The mean wind forces are given as follows:

$$
D_{s}=\frac{1}{2} \rho U^{2} B C_{D}, L_{s}=-\frac{1}{2} \rho U^{2} B C_{L}, M_{s}=\frac{1}{2} \rho U^{2} B^{2} C_{M},
$$

while the fluctuating buffeting forces are expressed in the subsequent form [24]:

$$
\begin{aligned}
D_{b} & =\frac{1}{2} \rho U^{2} B\left[2 C_{D} \chi_{D u} \frac{u}{U}+\left(C_{D}^{\prime}-C_{L}\right) \chi_{D w} \frac{w}{U}\right], \\
L_{b} & =-\frac{1}{2} \rho U^{2} B\left[2 C_{L} \chi_{L u} \frac{u}{U}+\left(C_{L}^{\prime}+C_{D}\right) \chi_{L w} \frac{w}{U}\right], \\
M_{b} & =\frac{1}{2} \rho U^{2} B^{2}\left(2 C_{M} \chi_{M u} \frac{u}{U}+C_{M}^{\prime} \chi_{M w} \frac{w}{U}\right),
\end{aligned}
$$

where $\rho$ is the still air density, $B$ is the deck width, $C_{D}=$ $C_{D}\left(\alpha_{s}\right), C_{L}=C_{L}\left(\alpha_{s}\right)$ and $C_{M}=C_{M}\left(\alpha_{s}\right)$ are the drag, lift and moment static wind coefficients, respectively, with their corresponding derivatives $C_{D}^{\prime}=\mathrm{d} C_{D} / \mathrm{d} \alpha_{s}, C_{L}^{\prime}=\mathrm{d} C_{L} / \mathrm{d} \alpha_{s}$ and $C_{M}^{\prime}=\mathrm{d} C_{M} / \mathrm{d} \alpha_{s}$, at the angle of static equilibrium $\alpha_{s}$. In the preceding equations, $\chi_{w}$ and $\chi_{u}$ are the frequency-dependent aerodynamic admittance functions. These functions are expressed in a complex form $\chi=\chi(K)=F+i G$, where $K=\omega B / U$ is the reduced frequency, $\omega$ is the frequency of the incoming wind fluctuations and $i=\sqrt{-1}$ is the imaginary unit. The self-excited forces are formulated as follows [25]:

$$
\begin{aligned}
& D_{s e}=\frac{1}{2} \rho U^{2} B( K P_{1}^{*} \frac{\dot{p}}{U}+K P_{2}^{*} \frac{B \dot{\alpha}}{U}+K^{2} P_{3}^{*} \alpha+K^{2} P_{4}^{*} \frac{p}{B} \\
&\left.+K P_{5}^{*} \frac{\dot{h}}{U}+K^{2} P_{6}^{*} \frac{h}{B}\right) \\
& L_{s e}=\frac{1}{2} \rho U^{2} B( K H_{1}^{*} \frac{\dot{h}}{U}+K H_{2}^{*} \frac{B \dot{\alpha}}{U}+K^{2} H_{3}^{*} \alpha+K^{2} H_{4}^{*} \frac{h}{B} \\
&\left.+K H_{5}^{*} \frac{\dot{p}}{U}+K^{2} H_{6}^{*} \frac{p}{B}\right) \\
& M_{s e}=\frac{1}{2} \rho U^{2} B^{2}\left(K A_{1}^{*} \frac{\dot{h}}{U}+K A_{2}^{*} \frac{B \dot{\alpha}}{U}+K^{2} A_{3}^{*} \alpha+K^{2} A_{4}^{*} \frac{h}{B}\right. \\
&\left.+K A_{5}^{*} \frac{\dot{p}}{U}+K^{2} A_{6}^{*} \frac{p}{B}\right)
\end{aligned}
$$

where $P^{*}=P^{*}(K), H^{*}=H^{*}(K)$ and $A^{*}=A^{*}(K)$ are the flutter derivatives, dependent on the reduced frequency
$K=\omega B / U$, where $\omega$ is the oscillation frequency. To transfor$\mathrm{m}$ the frequency-dependent terms in Eqs. (3) and (4) in a pure time-domain form, the rational approximation based on indicial functions is utilized herein [26]. The approach involves convolution of indicial response functions with the corresponding fluctuating velocities and displacements. For sake of brevity, the relations between the indicial functions and the aerodynamic admittance and flutter derivatives are not given. However, they can be found in an explicit form in [27] for the buffeting and in [28] for the self-excited forces.

\section{A novel turbulent Pseduo-3D vortex particle method}

In this section, initially, we revisit the governing equations of the VPM, 2D VTG and the laminar Pseudo-3D VPM. Based on these methods, in the sequel, we introduce the novel turbulent Pseudo-3D VPM.

\subsection{Vortex particle method}

The Navier-Stokes equations, written in a form describing the evolution of the vorticity field of a 2D incompressible flow at constant kinematic viscosity $v$, are expressed by the subsequent form [29]:

$$
\frac{\partial \omega_{u}}{\partial t}+(\boldsymbol{u} \cdot \nabla) \omega_{u}=v \nabla^{2} \omega_{u}
$$

where $\boldsymbol{u}$ is the fluid velocity vector obtained from the vorticity as $\omega_{u}=\omega_{u} \boldsymbol{e}_{n}=\nabla \times \boldsymbol{u}$, where $\boldsymbol{e}_{n}$ is the normal unit vector to the $2 \mathrm{D}$ plane. The continuity equation $\nabla \cdot \boldsymbol{u}=0$ assures a divergence-free vector field, which can be described uniquely as $\boldsymbol{u}=\nabla \times\left(\psi \boldsymbol{e}_{n}\right)+\boldsymbol{U}$. Here, $\psi=\psi(\boldsymbol{x})$ is the stream function and $\boldsymbol{U}$ is the free-stream velocity vector. The relation between the stream function and vorticity then results in a Poisson equation, which is expressed as follows:

$$
\nabla^{2} \psi=-\omega_{u} .
$$

In a domain $\mathcal{D}$, the Poisson equation can be solved for the vorticity using the Green's function as follows:

$$
\boldsymbol{u}(\boldsymbol{x})=\boldsymbol{U}-\frac{1}{2 \pi} \int_{\mathcal{D}} \frac{(\boldsymbol{x}-\boldsymbol{y}) \times \omega_{u}(\boldsymbol{y})}{|\boldsymbol{x}-\boldsymbol{y}|^{2}} \mathrm{~d} \boldsymbol{y},
$$

and is commonly referred to as the Biot-Savart relation. The boundary conditions of the velocity at the impermeable solid surface are expressed as follows [30]:

$$
\boldsymbol{u}\left(\boldsymbol{x}_{\mathcal{B}}\right) \cdot \boldsymbol{n}_{\mathcal{B}}=0, \quad \boldsymbol{u}\left(\boldsymbol{x}_{\mathcal{B}}\right) \cdot \boldsymbol{s}_{\mathcal{B}}=0
$$

where the $\boldsymbol{n}_{\mathcal{B}}$ and $\boldsymbol{s}_{\mathcal{B}}$ are the surface unit normal and tangential vectors of an immersed body $\mathcal{B}$, respectively. The total aerodynamic forces acting on an immersed object in the fluid can be obtained by integrating the surface pressure as follows:

$$
\boldsymbol{f}=\oint_{\mathcal{B}} p \boldsymbol{n}_{\mathcal{B}} \mathrm{d} \mathcal{B}
$$

where $p$ is the surface pressure, obtained from the vorticity (cf. [30]). 
In the VPM, the 2D vorticity field is discretized in $N_{p}$ number of particles at positions $\boldsymbol{x}_{p}$ as follows [31]:

$$
\omega_{u}(\boldsymbol{x}, t)=\sum_{p=1}^{N_{p}} \delta\left(\boldsymbol{x}-\boldsymbol{x}_{p}(t)\right) \Gamma_{p},
$$

where $\delta$ is the Dirac delta function. The circulation $\boldsymbol{\Gamma}_{p}=\Gamma_{p} \boldsymbol{e}_{n}$ carried by each vortex particle over the area of the particle $\mathcal{A}_{p}$, bounded by $\mathcal{S}$, is expressed by the following:

$$
\Gamma_{p}=\oint_{\mathcal{S}} \boldsymbol{u} \mathrm{d} \boldsymbol{s}=\int_{\mathcal{A}_{p}} \omega_{u} \mathrm{~d} \mathcal{A}_{p} .
$$

Substituting Eqs. (10) and (11) in Eq. (7), the discrete kinematic equation governing the fluid particles is given as follows:

$$
\boldsymbol{u}\left(\boldsymbol{x}_{p}\right)=\boldsymbol{U}-\sum_{p=1}^{N_{p}} \frac{\boldsymbol{e}_{n} \times\left(\boldsymbol{x}-\boldsymbol{x}_{p}\right)}{2 \pi\left|\boldsymbol{x}_{p}-\boldsymbol{x}\right|^{2}} \Gamma_{p}=\boldsymbol{U}-\sum_{p=1}^{N_{p}} \boldsymbol{K}\left(\boldsymbol{x}-\boldsymbol{x}_{p}\right) \Gamma_{p},
$$

where $\boldsymbol{K}$ is the velocity kernel. This kernel is replaced in the numerical implementation by a mollified velocity kernel $\boldsymbol{K}_{\epsilon}$ to account for the numerical instability in the denominator (cf. [30]).

The viscous vorticity transport equation is solved by the viscous splitting procedure, subsequently solving the convection step Eq. (12) and viscous step in Eq. (5). The stochastic random walk method developed by Chorin [32] is utilized for modeling of the diffusion, in which the particles are subjected to a random displacement with zero mean and $2 v \Delta t$ variance, $\Delta t$ being the simulation time-step. The solution of the Poisson equation (cf. Eq. (6)) for the kinetics is commonly based on a regularized mesh using the Vortex-In-Cell algorithms based on FFT solvers [33], in case of free-space or periodic boundary conditions. Herein, the fast $\mathrm{P}^{3} \mathrm{M}$ algorithm is utilized, implemented in the verified VPM platform VXflow [30]. The boundary element method is used for the discretization of the body geometry in piecewise linear panels. The boundary condition$\mathrm{s}$ in Eqs. (8) are imposed implicitly by the vorticity boundary conditions and utilizing Kelvin's circulation theorem as an additional constraint, which is given as follows [7]:

$$
\sum \Gamma=0 .
$$

\subsection{Velocity-based turbulence generation}

The 2D VTG for free-stream turbulence in the VPM was initially introduced by Prendergast [12, 13]. It represents a two-step procedure of sampling of a random velocity field on a ladder and subsequent conversion into circulation, which is concentrated in particles (cf. Fig. 1). The particles are then injected into a CFD domain, upstream of the section, at a constant rate. This simulates a free-stream turbulence with prescribed spectral characteristics.

In the first step, the sampled velocities are generated as twovariate $2 \mathrm{D}$ correlated stochastic processes, using the digital simulation method, which is based on the superposition of harmonic waves with a random phase [34]. The homogeneous twovariate random field $\boldsymbol{u}(\boldsymbol{x}, t)$ is spatially discretized as $P$ stochastic processes with zero mean as $\boldsymbol{u}(\boldsymbol{x}, t) \rightarrow\left\{\boldsymbol{u}_{1}(t), \boldsymbol{u}_{2}(t), \cdots, \boldsymbol{u}_{P}(t)\right\}$.
The statistical properties of the wind fluctuations are defined as a function of a circular frequency $\omega$ by the cross-PSD matrix $\boldsymbol{S}(\omega)$ with dimensions $2 P \times 2 P$, given by the following:

$$
\boldsymbol{S}(\omega)=\left[\begin{array}{ll}
\boldsymbol{S}_{u u}(\omega) & \boldsymbol{S}_{u w}(\omega) \\
\boldsymbol{S}_{w u}(\omega) & \boldsymbol{S}_{w w}(\omega)
\end{array}\right]
$$

where $S_{u u}, S_{w w}$ represent the cross-PSD matrices of longitudinal and vertical fluctuations. For the VTG, it is assumed that there is no correlation between the longitudinal and vertical fluctuations, i.e. $\boldsymbol{S}_{u w}=\boldsymbol{S}_{w u}=\mathbf{0}[13,14]$. The cross-PSD matrices are then formulated as follows [35]:

$$
S_{n_{j} n_{k}}(\omega)=\sqrt{S_{n}(\omega) S_{n}(\omega)} \operatorname{coh}_{j k}(\omega) \exp \left(-i \omega \theta_{j k}\right),
$$

where $c o h_{j k}$ is the coherence function and $\theta_{j k}$ is the angular phase shift, for $j, k \in\{1,2, \cdots P\}$ and $n \in\{u, w\}$. Since the boundary layer is not modeled in the VTG, the PSD $S_{n}$ is assumed to be independent of the height, i.e. $S_{n}(\omega, z)=S_{n}(\omega)$. The angular phase shift has a non-zero value for points along the direction of the mean velocity $U$, which coincides with $y$ direction (cf. Fig. 1). Based on Taylor's hypothesis, the angular phase shift can be computed as $\theta_{j k}=\Delta y_{j k} / U$, where $\Delta y_{j k}=y_{j}-y_{k}$. The Vickery's coherence function $\operatorname{coh}_{j k}(\omega)=$ $\operatorname{coh}\left(\Delta x_{j k}, \Delta y_{j k}, \Delta z_{j k} ; \omega\right)$ is used, which is defined as follows [35]:

$$
\operatorname{coh}_{j k}(\omega)=\exp \left(-\frac{\omega}{2 \pi} \frac{\sqrt{C_{x}^{2} \Delta x_{j k}^{2}+C_{y}^{2} \Delta y_{j k}^{2}+C_{z}^{2} \Delta z_{j k}^{2}}}{U}\right)
$$

where $C_{m}$ is the coherence coefficient and $\Delta m_{j k}=m_{j}-m_{k}$ is the separation distance between the points $j$ and $k$ for $m \in\{x, y, z\}$. In 2D, the span-wise distance amounts to $\Delta x=0$.

The cross-PSD matrix is a Hermitian matrix; hence, it can be decomposed as $\boldsymbol{S}(\omega)=\boldsymbol{H}^{*}(\omega) \boldsymbol{H}^{T}(\omega)$ using Cholesky decomposition, where $\boldsymbol{H}(\omega)$ is a lower triangular matrix, whose elements are complex functions of $\omega$. The superscripts "** and " $T$ " denote the conjugate complex and transposed operations, respectively. Each velocity component can be described as a Fourier-Stieltjes integral in the subsequent form:

$$
n_{j}(t)=\int_{-\infty}^{\infty} \exp (i \omega t) \mathrm{d} Z_{j}(\omega),
$$

for $n \in\{u, w\}$ over a random Fourier increment $d Z_{j}(\omega)$ and $j \in$ $\{1,2, \ldots, P\}$. Following the efficient representation introduced by Ding et al. [36], the integral can be discretized at constant incremental frequency $\Delta \omega$ as follows:

$n_{j}(t)=2 \sqrt{\Delta \omega} \Re\left\{\sum_{l=0}^{N_{f}-1} \sum_{k=1}^{P}\left|H_{j k}\left(\omega_{l}\right)\right| \exp \left[i \omega_{k l} t+i \phi_{k l}+i \theta_{j k}\left(\omega_{l}\right)\right]\right\}$,

where $\phi_{k l}$ is the random phase angle; $\omega_{l}=l \Delta \omega+\Delta \omega / 2$ is the single indexing frequency; $\omega_{k l}=l \Delta \omega+k / P \Delta \omega$ is the double indexing frequency for $l \in\left\{0,1, \ldots, N_{f}-1\right\}$ and $k \in\{1,2, \ldots, P\}$; $N_{f}$ is the number of discrete values for which the upper cutof$\mathrm{f}$ frequency is discretized as $\omega_{\max }=\Delta \omega N_{f}$. The phase angle 

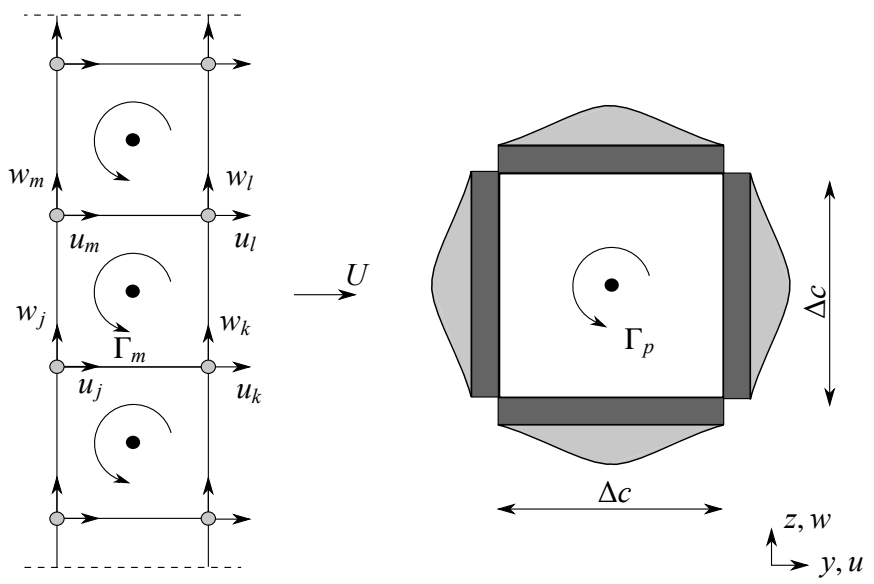

Figure 1: Conversion from nodal velocities on a ladder to particles. The particles are released upstream of the bridge section and are advected by the mean velocity (left). Velocity distribution of a vortex over the perimeter of a single quadratic cell (right): actual nonlinear distribution (light gray) and distribution assumed by nodal point approximation (dark gray).

can be computed from the real and imaginary part of the lower decomposed matrix as follows:

$$
\theta_{j k}\left(\omega_{l}\right)=\tan ^{-1}\left\{\frac{\mathfrak{I}\left[H_{j k}\left(\omega_{l}\right)\right]}{\mathfrak{R}\left[H_{j k}\left(\omega_{l}\right)\right]}\right\},
$$

where $\mathfrak{R}[\cdot]$ and $\mathfrak{J}[\cdot]$ represent the real and imaginary part, respectively. The Fourier transform is utilized for the numerical solution of Eq. (18) for the fluctuating velocities, which improves the efficiency significantly.

The second step in the VTG method is the conversion of the generated nodal velocity to a circulation, carried by vortex particles. The particles are then injected into the CFD domain at an inflow band. The circulation of an inflow particle is obtained by applying Eq. (11) for a quadratic cell (cf. Fig. 1) with size $\Delta c$ as follows:

$$
\Gamma_{p}=\int_{\mathcal{A}_{p}} \omega_{u} \mathrm{~d} \mathcal{A}_{p}=\left(\frac{\partial w}{\partial y}-\frac{\partial u}{\partial z}\right) \Delta c^{2} .
$$

Approximating the velocity distribution on the perimeter by the nodal velocities (cf. Fig. 1), the approximate circulation $\Gamma_{a}$ yields the following form $[13,37]$ :

$$
\Gamma_{a}=\frac{\Delta c}{2}\left[\left(w_{k}+w_{l}-w_{j}-w_{m}\right)-\left(u_{m}+u_{l}-u_{j}-u_{k}\right)\right] .
$$

For a single point vortex, the actual circulation $\Gamma_{p}$ is obtained from a nonlinear velocity distribution (light gray area in Fig. 1), while the approximated circulation $\Gamma_{a}$ is obtained from a linear velocity (dark gray area in Fig. 1). Hence, a correction factor of $\pi / 2$ should be applied in order to account for the nonlinear velocity distribution. The modeled vortex $\Gamma_{m}$ is then obtained as follows:

$$
\Gamma_{m}=\beta \Gamma_{p}=\beta \frac{\pi}{2} \Gamma_{a}
$$

where $\beta$ is taken as a correction factor. In case of a single vortex, $\beta$ amounts to 1 . However, for a grid of particles, rather than a ladder, this factor is reduced due to the cancellation of the errors the on the cell boundaries. This effect is referred to as "smudging of the vorticity" [13] and is briefly discussed in Sec. 4.3. The cell size $\Delta c$ is obtained using Taylor's hypothesis as $\Delta c=U \Delta p \Delta t$, where the particle release factor $\Delta p$ is defined to account for the difference between the simulation step $\Delta t$ and particle injection time-step $\Delta t_{p}=\Delta p \Delta t$. Injecting particles upstream in the domain introduces non-zero net circulation $\Gamma_{T}$ in the domain. Hence, the additional constraint for the boundary conditions, imposed by Kelvin circulation theorem (cf. Eq. (13)), needs to be modified as follows [13, 14]:

$$
\sum \Gamma=\sum \Gamma_{T}
$$

The conversion of ladder velocities to particles (cf. Eq. (21)) was validated on an actual 2D flow, represented by the numerical solution of the Navier-Stokes equations in [37]. Therein, the velocity field was recorded at a ladder, positioned in the wake of a bluff body. The flow was then reproduced using Eq. (21), which yielded satisfactory results.

The divergence-free condition on the velocity field, enforced by the continuity equation in the Navier-Stokes equations, does not apply to the generated velocity field (cf. [15]). In the vorticity transport equation (cf. Eq. 5), the divergence-free condition is implicit. Hence, there is a loss of kinetic energy when the kinematic constraints of the Navier-Stokes equations are applied to the generated velocity field. However, by extensive analyses of the turbulent flow characteristics, it was shown that the free-stream turbulence retains the statistical properties (cf. e.g. $[12-15])$.

\subsection{Laminar Pseudo-3D vortex particle method}

The 3D nature of the flow and structural behavior can severely affect the response of a line-like structure. The organized vortical structures in the fluid stretch and tilt due to the energy transfer between the turbulent eddies resulting in a complex 3D behavior of the aerodynamic load. The Pseudo-3D VPM attempts to include the 3D structural behavior while modeling the fluid in 2D planes (slices) along the span by employing the strip assumption (cf. Fig. 2). A 3D Finite Element model is used for the discretization of the structure, and the forces due to the fluid-structure interaction are applied at the finite element nodes. With this, several improvements can be made, despite the simplification of the 3D flow effects. These improvements include: (i) varying cross-sectional geometry along the structure, (ii) varying mean wind speed for each section and most importantly, (iii) the inclusion of an arbitrary number of structural modes which are necessary for adequate representation of 3D structural behavior [18]. The concept is straightforward: the force vector required for the external force required in Eq. (1) is assembled for $N_{s}$ number of slices as follows:

$$
f_{o}=\left[D_{1}, L_{1}, M_{1}, D_{2}, L_{2}, M_{2}, \ldots, D_{N_{s}}, L_{N_{s}}, M_{N_{s}}\right],
$$

where $D_{j}, L_{j}$ and $M_{j}$ for $j \in\left\{1,2, \cdots, N_{s}\right\}$ are the drag lift and moment force for the $j$-th slice. These forces are obtained from 
each slice by 2D CFD simulations, which are coupled by the structural model. For the representation of the structural model, a full- or reduced-mode order can be used. In case of laminar free-stream, it is reasonable to assume that the self-excited forces are fully correlated over one element, as noted by Scanlan [38]. This assumption was further examined in a numerical study [39], where it was observed that the 3D coherence effects in the wake have minor effect on the flutter derivatives for streamlined decks. However, the energy transfer between modes can severely affect the flutter limit [40], and thus, it is necessary to consider the $3 \mathrm{D}$ dynamic behavior of the structure.

\subsection{Turbulent Pseudo-3D vortex particle method with velocity- based turbulence generation}

In the case of free-stream turbulence, the $3 \mathrm{D}$ effects of the flow are perplexing. The turbulent Pseudo-3D VPM introduced herein attempts to replicate the incoming turbulent fluctuations in 2D, and enforce span-wise correlation in an empirical manner. The motivation is to obtain the dynamic response of a bridge deck due to an incoming turbulent wind, rather than to simulate free-stream coherent turbulent structures in detail. A two-variate $3 \mathrm{D}$ velocity field is generated on spatially correlated ladders, positioned upstream of each 2D fluid plane (cf. Fig. 3). The inflow particles are obtained at the center of each cell from the ladder by the VTG method, as described in Sec. 3.2.

Taylor's hypothesis of a frozen turbulence links the time and space correlation, by assuming that the turbulent field is advected entirely by the mean wind speed, that is $\boldsymbol{u}(x, y, z ; t)=$ $\boldsymbol{u}\left(x, y-U t, z ; t_{0}\right)$. In this section, we demonstrate that spanwise correlation of the circulation, and thus, velocity field remains correlated between slices, based on Taylor's hypothesis. The demonstration is conducted in two parts, in which: (i) it is shown that the circulation remains correlated in the span-wise direction, after the conversion from the nodal velocities at the ladder (cf. Eq. (21)); and (ii) it is shown that the correlation is retained as the particles are convected in the fluid domain.

Before we proceed, it is important to note that the following relations are only derived to demonstrate, in an analytical manner, that the span-wise correlation is maintained. In the implementation of the turbulent Pseudo-3D VPM based on the VTG, Eq. (21) is used to obtain the inflow particles after generating spatially correlated nodal velocities.

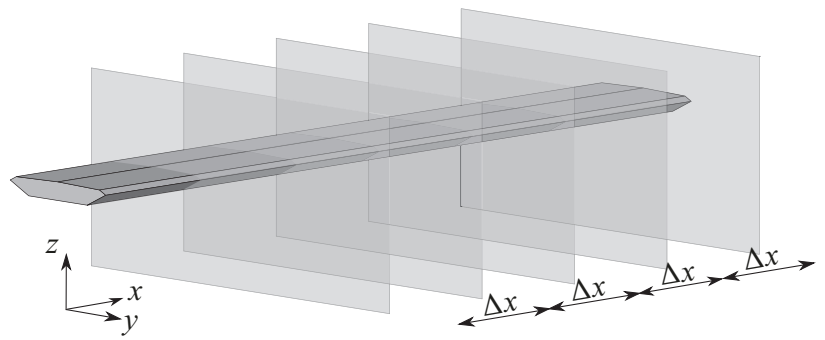

Figure 2: Discretization of the fluid domain on 2D planes (slices) along the span of a structure in the Pseudo-3D VPM.

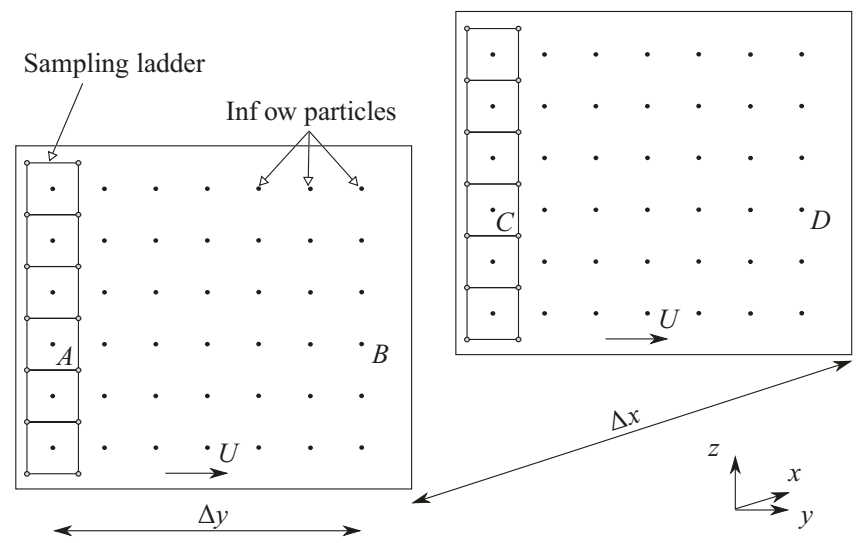

Figure 3: Sampling ladders and assumed position of inflow particles for two slices at distance $\Delta x$. The particles are released into the domain at the center of each cell of the ladder (inflow band) and are convected by the mean velocity $U$.

In the first part, initially the cross-covariance $R(\tau)$ of lag $\tau$ is defined for circulation $\Gamma$ at two points $X=\left(x_{1}, y_{1}, z_{1}\right)$ and $Y=$ $\left(x_{2}, y_{2}, z_{2}\right)$, located at the center of for any two cells. Utilizing Eq. (21) and assuming $\boldsymbol{S}_{u w}=\boldsymbol{S}_{w u}=\mathbf{0}$ (cf. Sec. 3.2) for the crossterms, the cross-covariance of the circulation at points $X$ and $Y$ is obtained as follows:

$$
\begin{aligned}
R_{\Gamma^{X} \Gamma^{Y}}(\tau)=E\left[\Gamma^{X}(t) \Gamma^{Y}(t+\tau)\right] & \\
=\frac{\Delta c^{2}}{4} E[ & {\left[w_{k}^{X}(t)+w_{l}^{X}(t)-w_{j}^{X}(t)-w_{m}^{X}(t)\right] } \\
& \times\left[w_{k}^{Y}(t+\tau)+w_{l}^{Y}(t+\tau)-w_{j}^{Y}(t+\tau)-w_{m}^{Y}(t+\tau)\right] \\
& +\left[u_{m}^{X}(t)+u_{l}^{X}(t)-u_{j}^{X}(t)-u_{k}^{X}(t)\right] \\
& \times\left[u_{m}^{Y}(t+\tau)+u_{l}^{Y}(t+\tau)-u_{j}^{Y}(t+\tau)-u_{k}^{Y}(t+\tau)\right],
\end{aligned}
$$

where $E[\cdot]$ is the expectation operator, while $n^{X}$ and $n^{Y}$ for $n \in\{u, w\}$ are the nodal velocities for the cells, in which the points $X$ and $Y$ are positioned, respectively. It is important to note that the circulations $\Gamma^{X}\left(x_{1}, y_{1}, z_{1} ; t\right)$ and $\Gamma^{Y}\left(x_{1}, y_{1}, z_{1} ; t\right)$ are not assigned to two particular particles, rather to each particle which is injected into the domain at time $t$ at position $X$ and $Y$. For wide sense stationary random processes, the WienerKhinchine relates the PSD $S(\omega)$ and the covariance $R(\tau)$ as follows:

$$
S(\omega)=\int_{-\infty}^{\infty} R(\tau) \exp (-i \omega \tau) \mathrm{d} \tau
$$

The covariance posses the unique symmetry $R_{a b}(\tau)=R_{b a}(-\tau)$. Therefore, the following relation can be obtained for $a=a(t)$ and $b=b(t)$ :

$$
S_{a b}(\omega)+S_{b a}(\omega)=S_{a b}(\omega)+S_{a b}^{*}(\omega)=2 \Re\left[S_{a b}(\omega)\right] .
$$

We obtain the cross-PSD of the circulation at points $X$ and $Y$ by applying the Wiener-Khinchin theorem (cf. Eq. (26)) on the cross-covariance (cf. Eq. (25)). Utilizing Eq. (27) and some 
basic operations, the subsequent form is obtained:

$$
\begin{array}{r}
S_{\Gamma^{X} \Gamma^{Y}}(\omega)=\frac{\Delta c^{2}}{4}\left\{S_{w_{k}^{X} w_{k}^{Y}}(\omega)+S_{w_{l}^{X}, w_{l}^{Y}}(\omega)+S_{w_{j}^{X} w_{j}^{Y}}(\omega)+S_{w_{m}^{X} w_{m}^{Y}}(\omega)\right. \\
+2 \mathfrak{R}\left[S_{w_{k}^{X} w_{l}^{Y}}(\omega)-S_{w_{k}^{X} w_{j}^{Y}}(\omega)-S_{w_{k}^{X} w_{m}^{Y}}(\omega)\right. \\
\left.\quad-S_{w_{l}^{X} w_{j}^{Y}}(\omega)-S_{w_{l}^{X} w_{m}^{Y}}(\omega)+S_{w_{j}^{X} w_{m}^{Y}}(\omega)\right] \\
+S_{u_{k}^{X} u_{k}^{Y}}(\omega)+S_{u_{l}^{X} u_{l}^{Y}}(\omega)+S_{u_{j}^{X} Y_{j}^{Y}}(\omega)+S_{u_{m}^{X} u_{m}^{Y}}(\omega) \\
+2 \mathfrak{R}\left[-S_{u_{k}^{X} u_{l}^{Y}}(\omega)+S_{u_{k}^{X} u_{j}^{Y}}(\omega)-S_{u_{k}^{X} u_{m}^{Y}}(\omega)\right. \\
\left.\left.\quad-S_{u_{l}^{X} u_{j}^{Y}}(\omega)+S_{u_{l}^{X} u_{m}^{Y}}(\omega)-S_{w_{j}^{X} u_{m}^{Y}}(\omega)\right]\right\} .
\end{array}
$$

Now, consider two points $A=\left(x_{o}, y_{o}, z_{o}\right)$ and $C=\left(x_{o}+\right.$ $\left.\Delta x, y_{o}, z_{o}\right)$ in the vicinity of two cells located at two separate slices (cf. Fig 3). To show that the circulation at these two points is correlated, the cross-PSD should not amount to zero, i.e. $S_{\Gamma^{A} \Gamma^{C}} \neq 0$. Usually, $\Delta x>\Delta c$; thus, we assume that the coherence between the nodal velocities for $A$ and the nodal velocities for $C$ can be approximated as follows:

$$
\operatorname{coh}(\Delta x, \Delta y, \Delta z ; \omega) \approx \operatorname{coh}(\Delta x, 0,0 ; \omega),
$$

where $\Delta y, \Delta z \in\{0, \Delta c\}$. Taking the preceding equation into account and that the prescribed PSD for the nodal velocities is independent of the height (cf. Sec. 3.2), we obtain the following relation by substituting Eq. (15) into Eq. (28):

$$
\begin{aligned}
S_{\Gamma^{A} \Gamma^{C}}(\omega)= & \frac{\Delta c^{2}}{4} S_{w}(\omega) \operatorname{coh}(\Delta x, 0,0 ; \omega) \\
& \times\{4+2 \mathfrak{R}[1-\exp (-i \omega \theta)-\exp (-i \omega \theta)- \\
& -\exp (-i \omega \theta) \exp (-i \omega \theta)+1]\} \\
& +\frac{\Delta c^{2}}{4} S_{u}(\omega) \operatorname{coh}(\Delta x, 0,0 ; \omega) \\
& \times\{4+2 \mathfrak{R}[-1+\exp (-i \omega \theta)-\exp (-i \omega \theta) \\
& -\exp (-i \omega \theta)+\exp (-i \omega \theta)-1]\},
\end{aligned}
$$

where $\theta=\Delta c / U$ is the angular phase shift. Utilizing only the real part of the Euler's formula, $\exp (-i \omega \theta)=\cos (\omega \theta)-$ $i \sin (\omega \theta)$, the cross-PSD of the circulation at points $A$ and $C$ yields the following:

$$
S_{\Gamma^{A} \Gamma^{C}}(\omega)=2 \Delta c^{2} \operatorname{coh}(\Delta x, 0,0 ; \omega) S_{w}(\omega)[1-\cos (\omega \theta)] .
$$

The preceding equation shows that the circulation at the points $A$ and $C$ is correlated as it includes the spatial coherence term. However, the parts due to space coherence and due to the PSD of the circulation $S_{\Gamma \Gamma}$ are not clearly distinguished. For this, Eq. (31) needs to be reduced in a form of Eq. (15). In order to do so, the PSD of the circulation $S_{\Gamma \Gamma}(\omega)=S_{\Gamma^{A} \Gamma^{A}}(\omega)=S_{\Gamma^{B} \Gamma^{B}}(\omega)$ is required.

The PSD $S_{\Gamma \Gamma}(\omega)$ is obtained by introducing Eq. (15) into Eq. (28). However, we will pose two more assumptions and obtain a "limit" form of $S_{\Gamma \Gamma}$, denoted as $\bar{S}_{\Gamma \Gamma}$. Strictly speaking, Taylor's hypothesis implies that $C_{y}=0$ [41], and assuming further that the product $\omega C_{z} \Delta c \ll U$, the coherence of the velocities in a single cell yields the following:

$$
\operatorname{coh}(0, \Delta y, \Delta z ; \omega) \approx 1,
$$

where $\Delta_{y}, \Delta_{z} \in\{0, \Delta c\}$. The limiting case of the PSD of the circulation is then obtained as follows:

$$
\begin{array}{r}
\bar{S}_{\Gamma \Gamma}(\omega)=\frac{\Delta c^{2}}{4} S_{w}(\omega)\{4+2 \mathfrak{R}[1-\exp (-i \omega \theta)-\exp (-i \omega \theta) \\
-\exp (-i \omega \theta)-\exp (-i \omega \theta)+1]\} \\
+\frac{\Delta c^{2}}{4} S_{u}(\omega)\{4+2 \mathfrak{R}[-1+\exp (-i \omega \theta)-\exp (-i \omega \theta) \\
-\exp (-i \omega \theta)+\exp (-i \omega \theta)-1]\},
\end{array}
$$

yielding the following concise form:

$$
\bar{S}_{\Gamma \Gamma}(\omega)=2 \Delta c^{2} S_{w w}[1-\cos (\omega \theta)] .
$$

By substituting for Eq. (34) into Eq. (31), the cross-PSD of the circulation for the points $A$ and $C$ is obtained as follows:

$$
S_{\Gamma^{A} \Gamma^{C}}(\omega)=\bar{S}_{\Gamma \Gamma}(\omega) \operatorname{coh}(\Delta x, 0,0 ; \omega) .
$$

The preceding equation clearly shows that the cross-PSD is constituted of two terms, the PSD $\bar{S}_{\Gamma \Gamma}(\omega)$ and the space coherence $\operatorname{coh}(\Delta x, 0,0 ; \omega)$. The two assumptions for the derivation of $\bar{S}_{\Gamma \Gamma}(\omega), C_{y}=0$ and especially Eq. (32), do not hold when the circulation is computed using the VTG method as in Eq. (21). Nevertheless, Eq. (34) is a limiting case $\left(\omega C_{z} \Delta c \rightarrow 0\right)$ and is given only to derive Eq. (35) from Eq. (31). In the VTG we use the "full" form for $S(\omega)$, obtained from Eq. (28) (which is omitted for the sake of brevity); therefore, if Eq. (29) holds, the cross-PSD term yields in the following:

$$
S_{\Gamma^{A} \Gamma^{C}}(\omega)=S_{\Gamma \Gamma}(\omega) \operatorname{coh}(\Delta x, 0,0 ; \omega) .
$$

The preceding equation shows that the circulation, and thus, the velocities for the VTG are correlated in the span-wise direction in the vicinity of the cells. Generally, the correlation is still maintained for even for small $\Delta x / \Delta c$ ratios as $S_{\Gamma^{A} \Gamma^{C}} \neq 0$; however, it cannot be shown in a concise form as Eq. (36). Regardless of the $\Delta x / \Delta c$ ratio, a partial loss of correlation is expected since the generated velocity field is not divergence-free. It is noteworthy to mention that Eq. (31) is dependent only on the PSD of the vertical fluctuations. This means that the span-wise correlation of the vertical fluctuating component will be governing. For cells which are additionally separated by vertical distance $\Delta z$, Eq. (36) will take similar form with the difference in the coherence $\operatorname{coh}(\Delta x, 0, \Delta z, \omega)$.

In the second part, we show that the cross-covariance of the circulation for the points $A=\left(x_{o}, y_{o}, z_{o}\right)$ and $C=\left(x_{o}+\right.$ $\left.\Delta x, y_{o}, z_{o}\right)$ at the ladder is similar as for the points $B=\left(x_{o}, y_{o}+\right.$ 
$\left.\Delta y, z_{o}\right)$ and $D=\left(x_{o}+\Delta x_{o}, y_{o}+\Delta y, z_{o}\right)$ downwind (cf. Fig. 3). Under Taylor's hypothesis, the along-wind distance $\Delta y$ relates to a time lag $\tau_{r}$ as $\tau_{r}=\Delta y / U$. Using the stationarity assumption, the cross-covariance $R(\tau)=R\left(\tau+\tau_{r}\right)$ can be expressed as follows:

$$
\begin{aligned}
R_{\Gamma^{A} \Gamma^{C}}(\tau) & =E\left[\Gamma\left(x_{o}, y_{o}, z_{o} ; t\right) \Gamma\left(x_{o}+\Delta x, y_{o}, z_{o} ; t+\tau\right)\right] \\
& =E\left[\Gamma\left(x_{o}, y_{o}, z_{o} ; t+\tau_{r}\right) \Gamma\left(x_{o}+\Delta x, y_{o}, z_{o} ; t+\tau_{r}+\tau\right)\right] \\
& =E\left[\Gamma\left(x_{o}, y_{o}+\Delta y, z_{o} ; t\right) \Gamma\left(x_{o}+\Delta x, y_{o}+\Delta y, z_{o} ; t+\tau\right)\right] \\
& =R_{\Gamma^{B} \Gamma^{D}}(\tau) .
\end{aligned}
$$

The latter equation shows, that the span-wise correlation of the circulation at the points $A$ and $C$ is retained in the along-wind direction.

Again, we stress that the previous relations were given to demonstrate that the span-wise correlation of the nodal velocities is maintained, once they are converted into particles. As shown in Eq. (37), this correlation is also maintained once the particles are advected with the mean flow. In the implementation, Eq. (21) is used or each cell after generating the correlated nodal velocities on the ladders positioned at multiple slices.

The idea of introducing empirically correlated free-stream turbulence to the Pseudo-3D VPM was utilized recently by Tolba and Morgenthal [20]. Therein, instead of using the VTG explained in Sec. 3.2, the PTG method was developed. In the latter method, the circulation of the particles is directly generated, instead of first generating a velocity field as in the VTG. It is important to stress out that the PTG method: (i) is only valid for isotropic turbulence; and (ii) considers only the one fluctuating velocity component for obtaining the circulation, which is derived based on the standard deviation instead of the PSD (see Eq. (37) from [20]). The VTG method for the free-stream turbulent fluctuations used herein is able to partially model anisotropic flow conditions, as discussed in Sec. 4.3. Furthermore, the velocity field is based on the PSDs of both fluctuating components. On the other hand, the span-wise correlation is enforced directly on the inflow particles in the PTG.

The energy transfer between turbulent eddies is different in 2D and 3D. However, the aim of the turbulent Pseudo-3D VPM is to model the large-scale eddies which carry the largest part of the turbulent energy and are critical for the aerodynamic response. The correlation in these eddies is higher than in the small eddies and for high Reynolds number, the advection is dominant. For fluid-structure interaction, a drawback of the turbulent Pseudo-3D VPM is the disregard of the 3D effects which result in increased correlation and reduced magnitude of the buffeting forces with respect to their 2D counterparts. Presently, the strip assumption is utilized, as it is difficult to empirically increase the span-wise correlation for the buffeting forces only, since they are nonlinearly coupled with the self-excited forces. Experimental studies (cf. e.g. [42]) showed that these effects are significant for wind tunnel tests. However, a recent study by Massaro and Graham [43] analytically shows that for bodies with large width-to-length ratios such as long-span bridges in full scale, the strip assumption is generally valid. It is note- worthy to mention that, in some of the recent semi-analytical nonlinear models (cf. e.g. [5, 44]), the manner of inclusion of the $3 \mathrm{D}$ effects is not fully elucidated yet.

In the atmospheric boundary layer the cross-PSD of the two fluctuation components can attain values $\boldsymbol{S}_{u w}(\omega) \neq \mathbf{0}$ for anisotropic turbulence, as measured by many experimental studies (cf. e.g. [35]) and described analytically for second-order structure of turbulence [45]. Nevertheless, the effect of $\boldsymbol{S}_{u w}(\omega)$ on the bridge response is commonly considered to be negligible relative to the overall uncertainty in the modeling of the wind field (cf. e.g. [46-48]). The cross-PSD term $S_{u w}(\omega)$ is neglected herein in order to maintain consistency with former studies using the VTG [13-15]. Moreover, the computational time for generation of wind time-histories is reduced significantly in this case as both fluctuation components can be generated independently, reducing the size of the cross-PSD matrix $S(\omega)$ by a factor of two. Presently, it is not established if the VTG method maintains the correlation between the two fluctuating components in $2 \mathrm{D}$. In case the correlation is preserved, the extension to Pseudo-3D would be straight-forward if it is assumed that the space-coherence is identical for both fluctuating components, i.e. $C_{m}=C_{m u}=C_{m w}$ for $m \in\{x, y, z\}$. In fact, Eq. (36) can be obtained in a similar manner. Otherwise, the coherence term could not be isolated as a product in Eq. (36) and the further expressions should be derived, which is out the of scope of this study. Demonstrating that the correlation between the fluctuating components is maintained, in 2D or Pseudo-3D, and studying its effect on slender structures with unconventional cross sections may represent a topic for further studies.

\section{Application}

In this section, we demonstrate the applicability of the Pseudo3D method. The turbulence statistics and span-wise correlation are first verified, followed by for buffeting and flutter analyses a long-span bridge. Moreover, the results from the CFD model are compared with the results from the LU model.

\subsection{Reference object}

Aerodynamically shaped, the Great Belt East suspension bridge extends over the Eastern Channel of the Great Belt with a total length of $2694 \mathrm{~m}$ and the main span of $1624 \mathrm{~m}$ (cf. Fig. 4, left). The deck is a $31 \mathrm{~m}$ wide steel box girder with a depth of $4.4 \mathrm{~m}$ (cf. Fig. 4, right). Specific dimensions of the deck can be found in [49] for the model H9.1, without the additional equipment such as barriers and fairings. The lack of detailed modeling of the additional equipment herein is in order to reduce the computational load, since very fine discretization is required to resolve to small turbulent scales, thin boundary layer and complex flow structures in the region of the deck details. Nevertheless, the ability of the VPM to resolve such flow features has been shown in former studies (cf. e.g. [9, 18, 50]). Therefore, the application of the presented method could be directly extended to bridge decks including traffic barriers and other devices without any further modification.

The girder mass per unit length including cables is taken as $m=22.74 \mathrm{t} / \mathrm{m}$ and the mass moment of inertia as $I=2.47 \times 10^{3}$ 


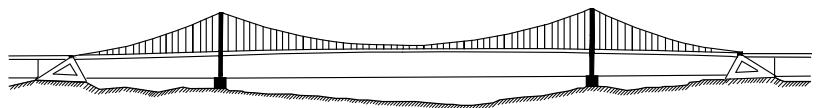

$\stackrel{535 \mathrm{~m}}{\longleftrightarrow} \longleftarrow \stackrel{1624 \mathrm{~m} \longrightarrow 535 \mathrm{~m}}{\longleftrightarrow}$

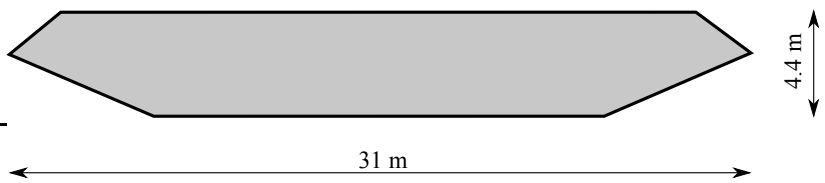

Figure 4: Schematic of the reference object: elevation of the Great Belt suspension bridge (left) and cross-section (right). $\mathrm{tm}^{2} / \mathrm{m}$. A finite element model is build of the bridge, from which the deck modes are extracted for the 3D analysis. The wind forces are applied to the deck only, without a loss of generality, and the deck is discretized on 51 finite elements. For the Pseudo-3D analyses, a 2D slice is positioned at the center of each element. The dynamic properties of the model are calibrated against the ones presented in [51]. The first lateral, vertical and torsional frequencies are achieved as $f_{p}=0.052$ $\mathrm{Hz}, f_{h}=0.100 \mathrm{~Hz}$ and $f_{\alpha}=0.278 \mathrm{~Hz}$, respectively. A total of 22 modes with a maximum frequency of $0.5 \mathrm{~Hz}$ are used for the 3D analyses, of which 7 lateral, 12 vertical and 3 torsional. The modal damping ratio is set as $0.5 \%$.

\subsection{Aerodynamic coefficients}

In the CFD simulations, the section is discretized by 250 panels with an average length amounting to $\Delta l / B=8.075 \times 10^{-3}$, from which the reduced time step is computed as $\Delta s=2 \Delta l / B=1.65 \times 10^{-2}$, where $s=t B / U$ is the non-dimensional

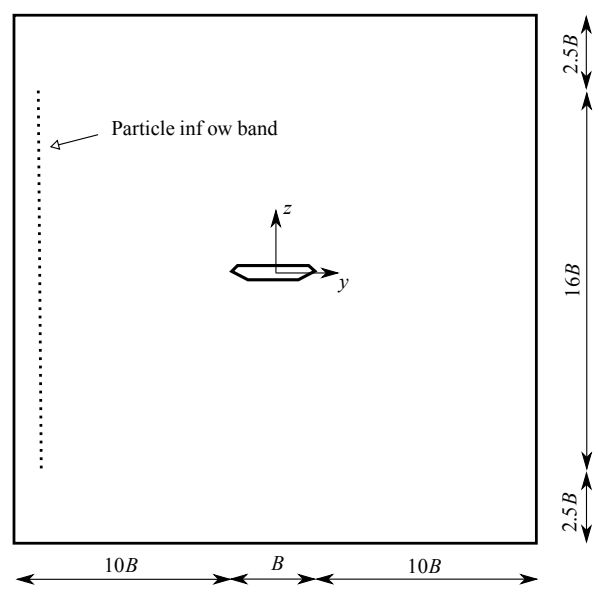

Figure 5: Dimensions of the selected fluid domain for a single slice along with the particle inflow band positioned at $y / B=-10$.

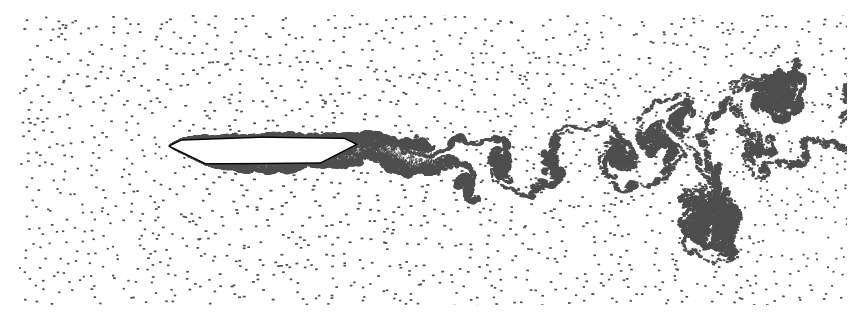

Figure 6: Instantaneous particle map of a turbulent flow past the Great Belt section. time. The Reynolds number $\operatorname{Re}=U B / v$ is set as $1.03 \times 10^{5}$. Accounting for a constant turbulent field in the oscillation region with sufficient wake, the selected domain is depicted in Fig. 5.

Aerodynamic coefficients including static wind coefficients, fluter derivatives and aerodynamic admittance functions are given herein for the CFD model. These coefficients represent the necessary input for the LU model used to verify the results of the aeroelastic analyses. In Fig. 6, an instantaneous snapshot of a particle map is shown for a section immersed in turbulent flow. The static wind coefficients (cf. Fig. 7) are obtained including free-stream turbulence, with turbulent properties defined in the following section. A fairly linear trend can be noted for $\pm 4 \mathrm{deg}$ for the lift $C_{L}$ and moment $C_{M}$ coefficients. Since the LU model is a linear model, it can be assumed that the quasi-steady behavior can be well represented by this model for this range. The flutter derivatives utilized herein are taken from [16], obtained using the forced vibration method in a smooth flow with the same numerical parameters as the present study. Figure 8 depicts the flutter derivatives with respect to the reduced velocity $V_{r}$, which are further compared to the available experimental results [49], yielding satisfactory results. Quasisteady values are used for the $P_{j}^{*}$ for $j \in\{1,2, \ldots, 6\}$ and $H_{j}^{*}$, $A_{j}^{*}$ for $j \in\{5,6\}$ derivatives. Additionally, the flutter derivatives are obtained for a varying positive angle of incident, in order to perform the flutter analysis at the angle of static equilibrium. As an example, the $A_{2}^{*}$ derivative is shown in Fig. 9. A change from negative to a positive value of $A_{2}^{*}$ is noted at angle amounting to $\alpha=4 \mathrm{deg}$, indicating a change of mechanism from coupled to single degree of freedom torsional flutter. This is further discussed in Sec. 4.4.

The aerodynamic admittance functions are computed using the spectral method $[14,15]$ with turbulent properties defined subsequent section. Figure 10 shows the square modulus of

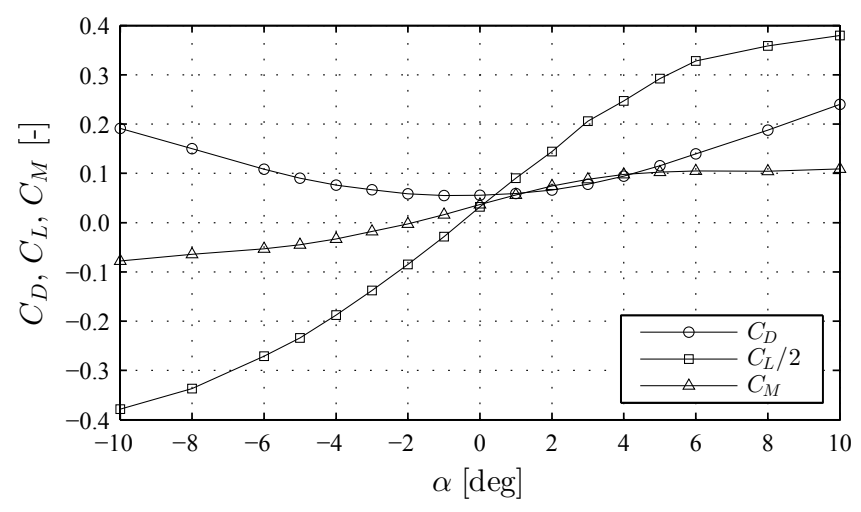

Figure 7: Static wind coefficients. 

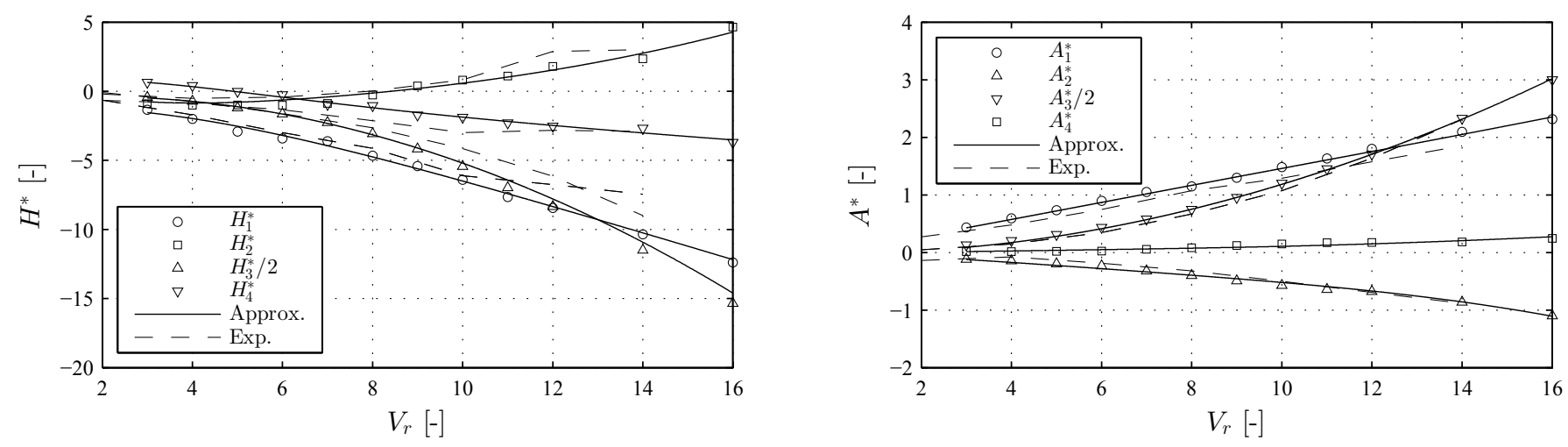

Figure 8: Flutter derivatives and their rational approximation (Approx.) at zero degree angle of incident wind. The experimental values are obtained from [49].

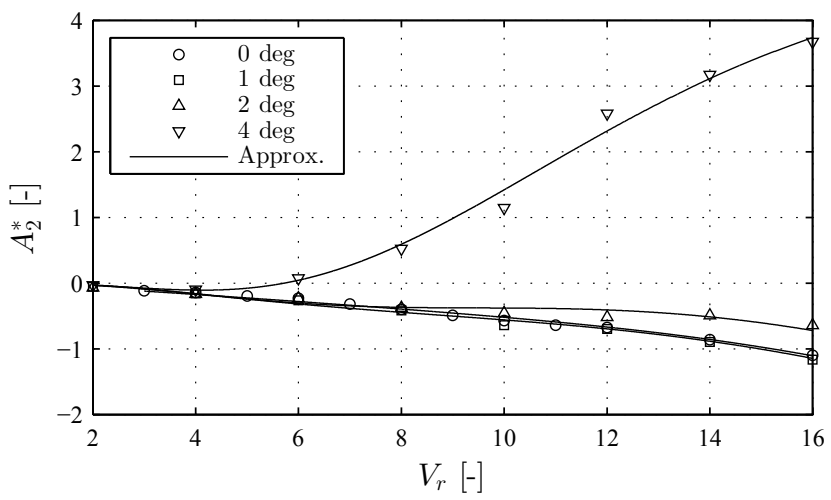

Figure 9: Flutter derivative $A_{2}^{*}$ for various static angles of attack $\alpha_{s}$.

the lift and moment aerodynamic admittance functions. With the spectral method, only the square modulus of the admittance can be obtained; hence, it is assumed that $\chi_{L}=\chi_{L u}=\chi_{L w}$ and $\chi_{M}=\chi_{M u}=\chi_{M w}$. To perform the rational approximation, the imaginary part of the complex admittance $\chi=F+i G$ is assumed to be the same as the one from the complex approximation of the Sears' admittance [52], while the real part is computed based on the squared modulus. Furthermore, a cutoff at $K=\pi$ is set for the rational approximation in order to separate the incident part of the admittance from the vortex shedding. It should be noted that spectral determination of the aerodynamic admittance is a common practice in experimental tests (cf. e.g. $[53,54])$. The admittance of the drag force is taken as unitary, that is, $\chi_{D}=\chi_{D u}=\chi_{D w}=1$.

\subsection{Flow verification}

The 2D free-stream turbulent statistics for the VTG method were assessed in [14], based on the ESDU spectra. Herein, the 2D anisotropic turbulent statistics, based on the von Kármán PSD, are investigated briefly. This is followed by a verification of the empirical span-wise correlation between slices. The von
Kármán PSD function is given as follows [35]:

$$
\begin{aligned}
\frac{f S_{u u}(f)}{\sigma_{u}^{2}} & =\frac{4 f L_{u} / U}{\left(1+70.8\left(f L_{u} / U\right)^{2}\right)^{5 / 6}}, \\
\frac{f S_{w w}(f)}{\sigma_{w}^{2}} & =\frac{4 f L_{w} / U\left(1+755.2\left(f L_{w} / U\right)^{2}\right)}{\left(1+283.2\left(f L_{w} / U\right)^{2}\right)^{11 / 6}},
\end{aligned}
$$

where $L_{j}$ is the turbulent length scale and $\sigma_{i}$ is the standard deviation of the fluctuations for $j \in\{u, w\}$. The turbulence intensity $I_{j}=\sigma_{j} / U$ is set as $I_{u}=11 \%$ and $I_{w}=6 \%$ for the lateral and vertical fluctuations, respectively, with a mean wind speed $U=30 \mathrm{~m} / \mathrm{s}$. The turbulent length scales amount to $L_{u}=108 \mathrm{~m}$ and $L_{w}=30 \mathrm{~m}$, while the coherence coefficients are set as $C_{x}=5$, $C_{y}=3$ and $C_{z}=10$. All of the analyses are conducted for a dimensional time of $t=700 \mathrm{~s}$. The height of the particle release ladder is set to $H_{p}=16 B$ (cf. Fig. 5). Before investigating turbulent properties, we define the generated nodal velocity on the ladder as "generated" velocity. The velocity, which is tracked down in the domain during the simulation, is defined as "simulated" velocity. The particle release factor is taken as $\Delta p=4$. This resulted in 240 particles per release step with a total of $N_{f}=5.04 \times 10^{3}$ discrete frequencies. Most of the generated turbulent energy converged for the selected discretization of the PSD, resulting in a good agreement between the prescribed and generated turbulence intensities.

Figure 11 shows the results of a parametric study of the correction factor $\beta$ (cf. Eq. (22)) for a single slice. In this case, it can be seen that for $\beta=0.65$ (i.e. $\beta \pi / 2 \rightarrow 1$ ) the vertical turbulence intensity converges to the prescribed value, while for the lateral component the turbulence intensity is found to be reduced to $70 \%$ of the prescribed for the point at $(0,0)$. Generally, $\beta$ can take values from 0.6 up to 1 , depending on the PSD function used and the hight of the particle ladder. In case of isotropic turbulence, the turbulence intensity for both components converges for values of $\beta$ approximately amounting to $\beta=0.7$. The reason for the factor $\beta$ not having a value of one is due to the "smudging" effect of the vortices, as the circulation $\Gamma_{p}$ only for a single cell, tends to the approximated one $\Gamma_{a}$. This was shown by Prendergast [13], who used $N_{p} \times N_{p}$ grid to replicate a circular vortex and concluded that in case the grid 

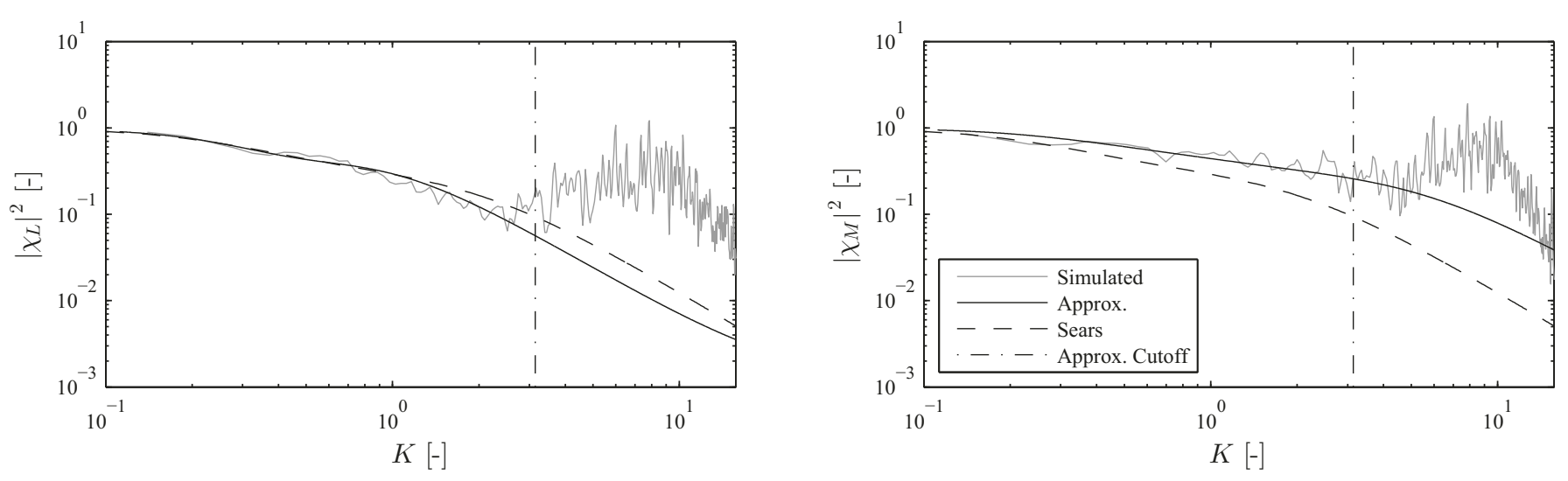

Figure 10: Squared modulus of the aerodynamic admittance functions and their rational approximation (Approx.) compared to the Sears' admittance [52]. The cutoff frequency (Approx. Cutoff) is chosen as $K=\pi$ in order to separate the incident part of the wind fluctuations from the vortex shedding for the rational approximation.
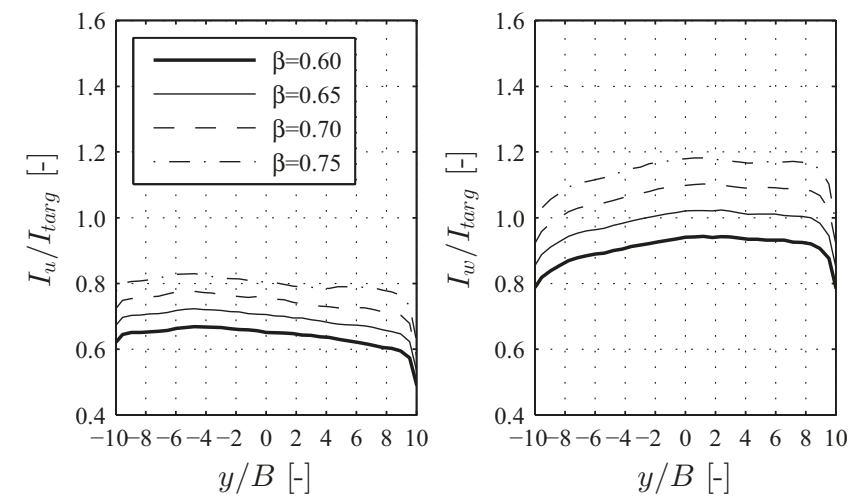

Figure 11: Parametric study for the factor $\beta$, accounting for the nonlinear distribution of the velocities on the perimeter of the sampling ladder. For this study, $\beta=0.65$ is selected.

is larger than $3 \times 3$, the factor $\beta \pi / 2 \rightarrow 1$. Furthermore, Chawdhury and Morgenthal in [37] used the method for conversion of ladder velocities into particles to reproduce a wake behind a bluff body where the velocities sampled on the ladder are obtained from the solution of the Navier-Stokes equation. The factor $\beta$ is reported to be around 0.6 to match the reproduced flow turbulence properties. Similar discrepancies for anisotropic turbulence properties were observed in [14]. The loss of the anisotropic properties is believed to be a result of the converting of the wind velocities into particles (cf. Eq. (21)), since the generated velocity field is not divergence-free [15].

The PSD of the simulated signal at point $(0,0)$ in the domain is computed using Welch's method with $50 \%$ overlap and is compared to the prescribed one in Fig. 12. The spectral distribution is retained for the PSD of the longitudinal velocity component; however, a constant reduction is noted. The PSD of the vertical velocity component corresponds well with the prescribed one. A distinctive cutoff frequency is present for both velocity components, where the longitudinal energy decreases while the vertical increases. This is a result of the Nyquist sam- pling rate of the generated velocity, that is, $f_{\text {gen,max }}=\Delta p f_{\text {sim, } \text { max }}$, where $f_{\text {sim,max }}$ is the Nyquist sampling rate of the simulated velocity. For $U=30 \mathrm{~m} / \mathrm{s}, f_{\text {gen, } \text { max }}$ amounts to $7.34 \mathrm{~Hz}$.

Taylor's hypothesis is the basis for the span-wise correlation, as established in Sec. 3.4. To test this hypothesis, the temporal $r_{t}=r(\tau)$ and spatial $r_{y}=r(y)$ correlation coefficients are given for the $2 \mathrm{D}$ domain in Fig. 13. The temporal correlation coefficient is obtained from the velocity fluctuations at $(0,0)$ in the domain, while the spatial correlation coefficient is computed from the velocity fluctuations at points along the centerline $z / B=0$. As observed from Fig. 13, $r_{y}$ and $r_{t}$ correspond well. Thus, Taylor's hypothesis is mostly valid for this case.

The correlation of wind fluctuations between slices in the turbulent Pseudo-3D VPM represents an essential part. Thus, we study the span-wise correlation on a test-case with $N_{s}=6$ slices at uniform lateral distance $\Delta x=5 \mathrm{~m}$, initially without a section in the domain. Figure 14 depicts the span-wise correlation coefficient $r_{x}=r(x)$ for of the first slice with respect to the rest of the slices for the simulated velocity along the center of the fluid domain, $z / B=0$ (cf. Fig. 5). The span-wise correlation coefficient of the simulated velocities is further compared to the one from the generated fluctuations. In case of the longitudinal fluctuations, an immediate reduction is observed near the particle inflow band $(y / B=-7.5)$. The behavior of $r_{w y}$ yielded in good correspondence with the correlation of the generated wind fluctuations. This is in line with the conjuncture we made in Sec. 3.4 (cf. Eq. (31)), that the vertical fluctuations govern the span-wise correlation. Nevertheless, both of the components maintain the exponential shape of decay. It can be also observed that the correlation does not reduce significantly downwind, showing the validity of Taylor's hypothesis. Therefore, the conversion from the nodal velocities into particle strength is the probable cause for loss of correlation in the longitudinal direction. Another effect which may contribute to this loss is the viscosity of the fluid. Once particles are injected into the domain, there is no interaction, and thus, there is a loss of correlation due to diffusion. However, this effect is not significant for high Reynolds numbers due to the minor role of the 

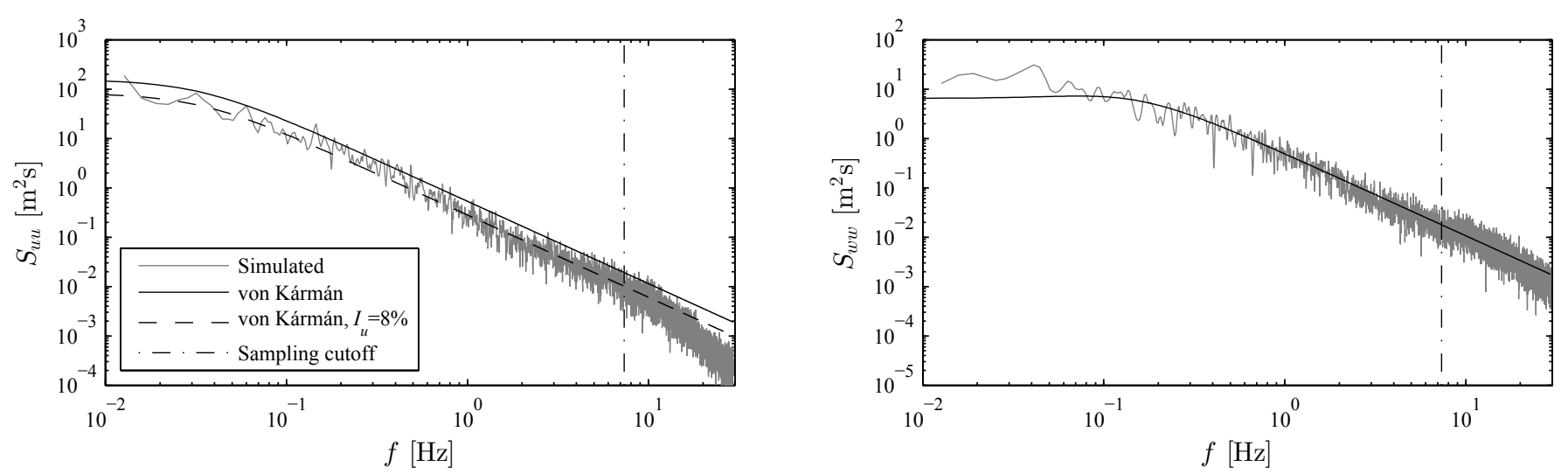

Figure 12: PSD for the horizontal $S_{u u}$ (left) and vertical $S_{w w}$ (right) simulated fluctuating component at $(0,0)$ in the domain. The sampling cutoff represents a Nyquist sampling rate $f_{\text {gen,max }}$ for particle release factor $\Delta p=4$.

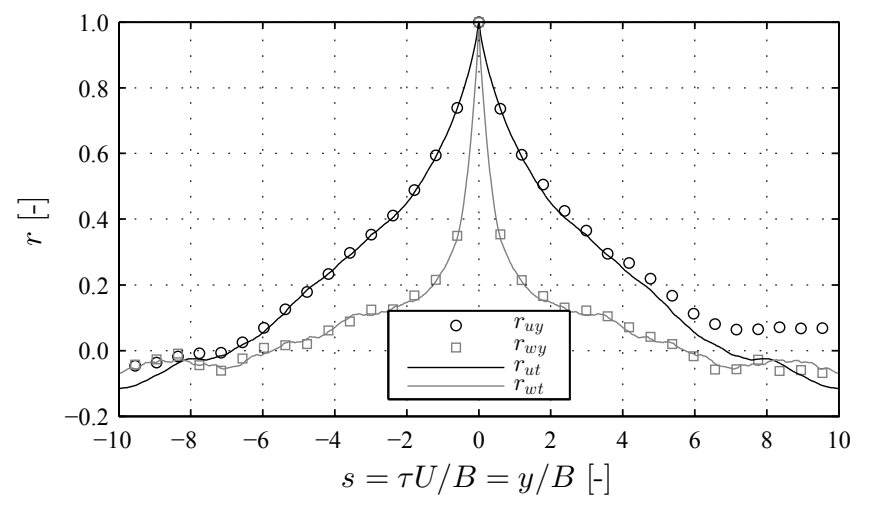

Figure 13: Spatial $r_{y}=r(y)$ and temporal $r_{t}=r(\tau)$ correlation coefficients. The temporal correlation coefficient is obtained from the fluctuating velocities at point $(0,0)$, while the spatial one from velocity fluctuations at points along the centerline $z / B=0$.

viscous forces, hence the validity of Taylor's hypothesis.

To study the span-wise correlation of the buffeting forces, we add a stationary section within the domain for the previous test-case. The correlation coefficient of the forces is slightly higher than the wind fluctuations (cf. Fig. 15). Although the increased correlation of the buffeting forces is predominately due to the 3D effects, Larose [42] notes the distortion of the freestream turbulence by the deck, i.e. the "physical averaging of the wind fluctuations" as a factor for the increased span-wise correlation. In order to further illuminate this point and the reason for loss of correlation in the lateral fluctuations, the magnitude squared coherence is investigated for the wind fluctuations and forces between the first and third slice (cf. Fig. 16). For the vertical fluctuations, it can be observed that the agreement is good with the prescribed Vickery's coherence function (cf. Eq. (16)). The shape of the coherence for the longitudinal fluctuation component is similar; however, for certain frequencies, there is an underestimation with respect to the prescribed coherence. Nevertheless, the coherence tends asymptotically towards one, indicating that the large eddies carrying most of the turbulent energy are correlated. The lift and moment coherence mostly coincides with the one of the vertical fluctuations. Thus, it is not clear that the increment in the span-wise correlation coefficient of the forces with respect to the wind fluctuations is due to the physical averaging of the wind or vortex shedding. Additionally, noise may affect the correlation coefficient; therefore, the coherence is a more reliable measure. It is noteworthy to mention that, the coherence within the CFD domain is not reported in the studies dealing with bridge vibration due to turbulent wind (cf. e.g. [20, 55]).

\subsection{Flutter analysis}

Multimode flutter analysis is conducted using the modal properties described in Sec. 4.1. The critical flutter velocity $U_{c r}$ is determined using the laminar Pseudo-3D VPM and the LU model in the time domain. Figure 17 depicts the time-histories of the vertical and torsional displacements for the LU and the CFD model, for velocities below and at critical flutter velocity. Furthermore, the displacements for a divergent velocity for the LU model are given. The static displacements due to the mean wind velocity for the LU model are omitted in the time histories, in order to distinctively show the difference between the damped, critical and divergent oscillations. By examining the time histories at the critical flutter velocity corresponding to the CFD model, it can be observed that the section is initially displaced due to the mean velocity, which is followed by a coupled divergent oscillation. As the amplitude increases, the mean of the vertical displacements tends to zero, i.e. the section "drops" and oscillates about the initial position. This type of instability is sometimes referred to as stall flutter and is typically characterized by a large flow separation. The same kind of behavior for the bare deck of the Great Belt section is reported in $[16,56]$ in 2D. LCO with constant amplitudes are evident in the post-flutter range (approx. $t>300 \mathrm{~s}$ ) for the CFD model. The LU model is unable to capture the LCO since it is a linear model, whereas the unsteady nonlinearity governs the motion amplitudes for the post-flutter regime. To the best of the authors' knowledge, amplitudes in the post-flutter regime from experiments have not been reported in the literature for the Great Belt 

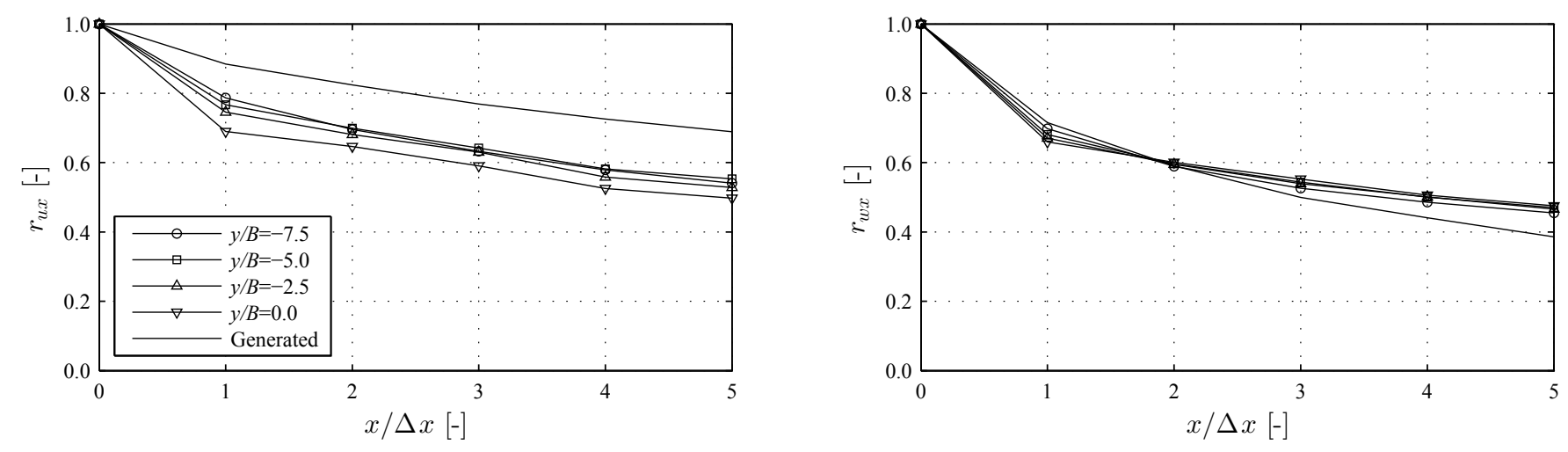

Figure 14: Span-wise correlation coefficient $r_{x}$ between slices of the generated and simulated wind velocity at different locations along the centerline $z / B=0$ for the selected test case: $r_{u x}$ (left) and $r_{w x}$ (right). The test-case is without a section in the domain.

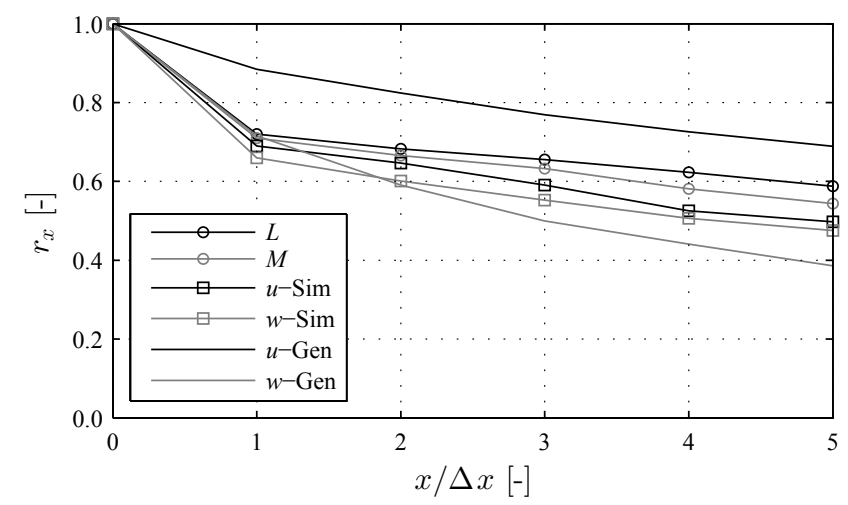

Figure 15: Span-wise correlation coefficient $r_{x}$ between slices of the deck forces, generated (Gen) and simulated (Sim) wind fluctuating velocities at center point $(0,0)$ for the selected test case.The test-cases are without and with a section in the domain for the wind fluctuations and forces, respectively.

bridge. Due to the lack of reference data, the amplitudes obtained here should be taken with a proper consideration as they can be over- or underestimated. Generally, the VPM is capable of accurately modeling the post-flutter behavior and matching the LCO amplitudes, as it is recently validated in [57] with experimental results for an energy harvester. Figure 18 shows the instantaneous particle map at the peak of the positive rotation (nose-up) in the LCO. The contribution of the first vertical and torsional modes are evident. Since the self-excited forces are usually considered as fully correlated, the utility of the Pseudo3D VPM for the determination of the instability threshold in multimode flutter analysis is prominent.

The critical flutter velocity $U_{c r}$ appeared to be sensitive for positive angles of incidence (nose-up). Accordingly, the flutter derivatives are determined at a different angle of incidence, as noted previously in Sec. 4.1. From the $A_{2}^{*}$ derivative depicted in Fig. 9, it can be observed that the flutter regime changes from coupled to torsional flutter at static angle amounting to $\alpha=4 \mathrm{deg}$. Rotating the section increases the width over depth sectional ratio, and thus, the length of the separation bubble.
These are the key factors for the flutter regime, as it has been tested experimentally for the fundamental case on rectangular cylinders [58]. Therefore, a nonlinear aerostatic analysis is performed in case of the LU model to obtain the angle of static equilibrium $\alpha_{s}$ as a function of the wind speed. Chen and Kareem [59] highlight that performing flutter analysis with respect to the angle $\alpha_{s}$ can be the governing case in certain occasions. Figure 19 shows the critical flutter velocity for the CFD and LU models. In case of the latter, the critical velocity is obtained for various values of the angle $\alpha$. The CFD model resulted in lower instability limit with respect to the LU model at the angle of static equilibrium $\alpha_{s}$, with a difference of $5.5 \%$. It should be noted that, the critical flutter limit at the angle of static equilibrium in case of the LU model $\left(U_{c r}\right.$-LU for $\left.\alpha=\alpha_{s}\right)$ is obtained by using linear interpolation of the critical velocity. Alternatively, the instability threshold for the LU model can be determined directly from the flutter analysis using interpolated rational coefficients for the indical functions at the angle of static equilibrium, as noted in [59]. Nevertheless, in this case, the flutter derivatives are obtained at an increment of 1 degree, and thus, the deviations are expected to be small for the simple approach used herein. The angle at static equilibrium for the CFD model is obtained by averaging the rotations at a velocity that is $1 \mathrm{~m} / \mathrm{s}$ less than the critical one. Since critical flutter velocity for the CFD model lies on the $\alpha_{s}-U$ curve, it can be further concluded that the nonlinear aerostatic analysis of the LU model yielded good correspondence with the CFD model.

To validate the laminar Pseudo-3D method, the flutter limit is computed using the LU model and the experimental flutter derivatives at angle amounting to $\alpha=0$. Since the $A_{4}^{*}$ flutter derivative is not determined from the experiments reported in Reinholdt et al. [49], the corresponding value from the CFD analyses is used. Good correspondence can be observed between the critical velocities for the CFD and experimental flutter derivatives with a difference of $2.5 \%$. Moreover, Larsen [51] reported an instability threshold amounting to $U_{c r} \approx 72 \mathrm{~m} / \mathrm{s}$ from free-vibration experimental tests for a taut strip model, which is similar to the results from the Pseudo-3D analysis. For free-vibration tests, the angle of attack is automatically corre- 

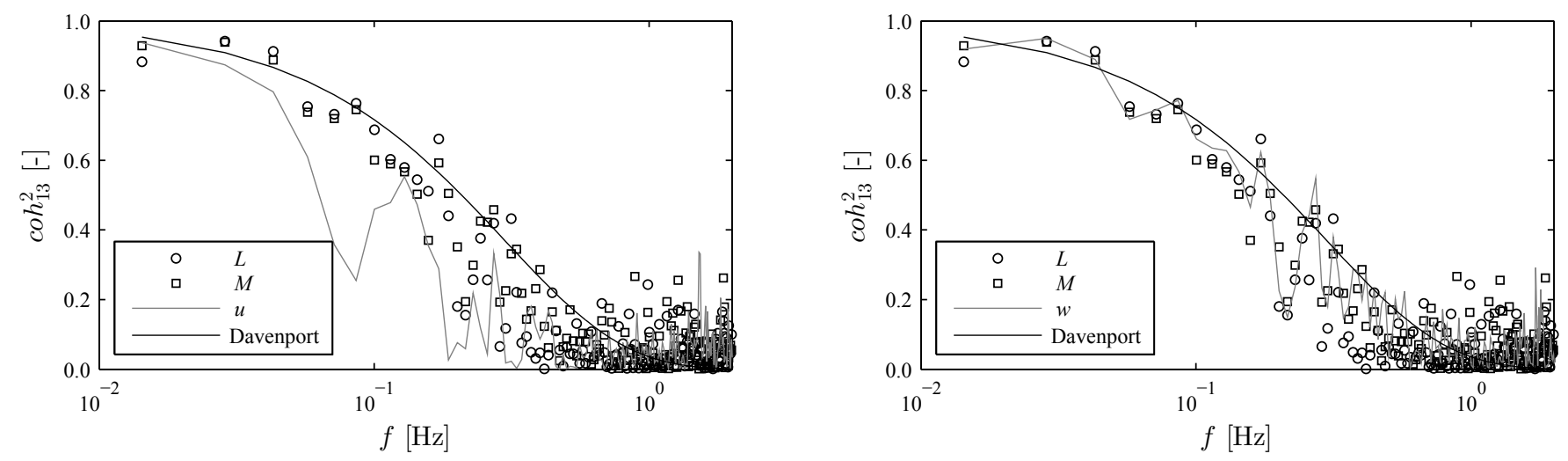

Figure 16: Magnitude-squared coherence of the forces and wind fluctuations for the first $(x / \Delta x=0)$ and third slice $(x / \Delta x=2)$ at $(0,0)$ in the domain. The test-cases are without and with a section in the domain for the wind fluctuations and forces, respectively.
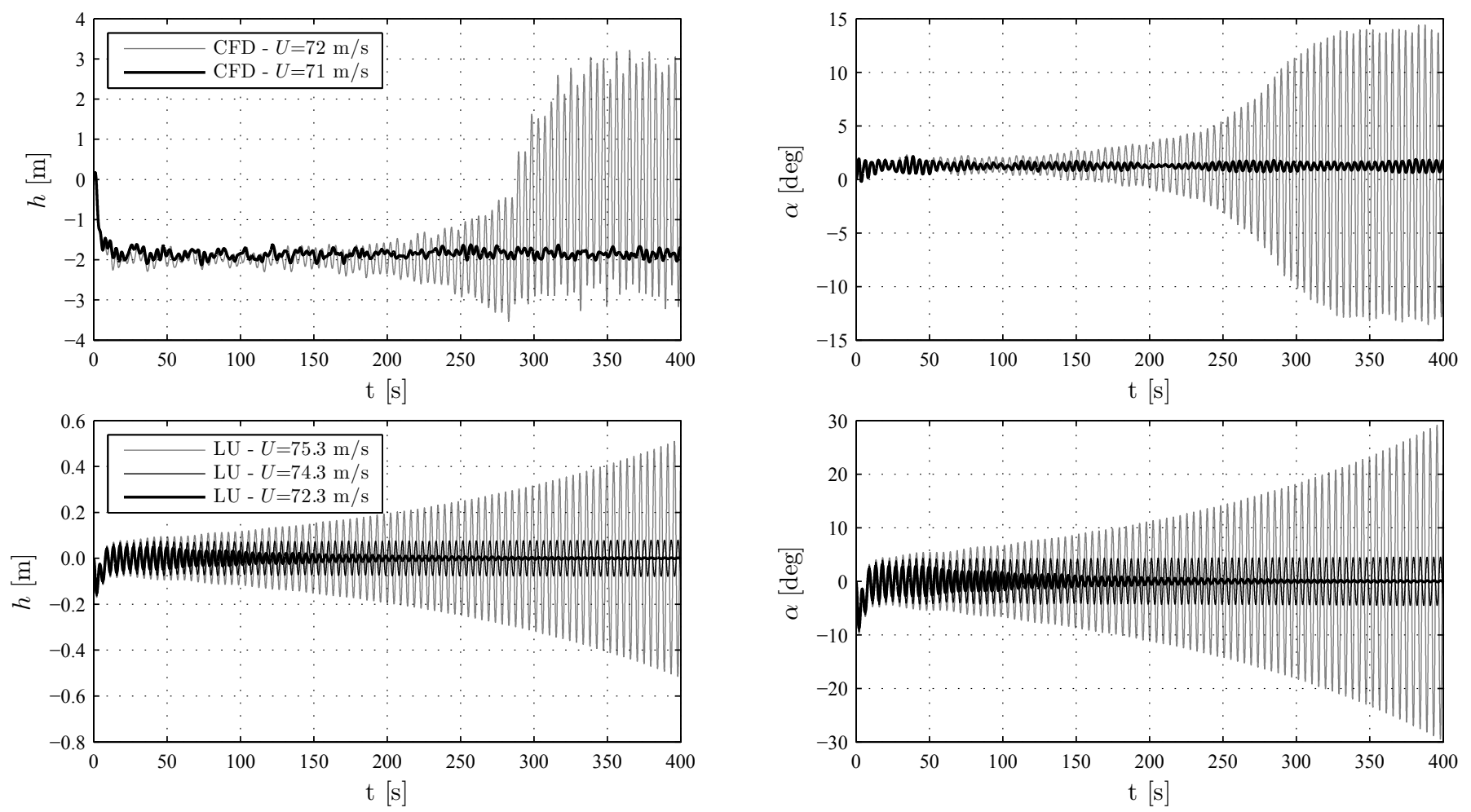

Figure 17: Time-histories of the vertical displacements (top-left) and rotation (top-right) for the damped $(U=71 \mathrm{~m} / \mathrm{s})$ and LCO $(U=72 \mathrm{~m} / \mathrm{s})$ case for the CFD model under laminar flow. Damped $(U=72.3 \mathrm{~m} / \mathrm{s})$, critical $(U=74.3 \mathrm{~m} / \mathrm{s})$ and divergent $(U=75.3 \mathrm{~m} / \mathrm{s})$ oscillations for vertical displacements (bottom-left) and rotation (bottom-right) obtained using the LU model at $\alpha=2 \mathrm{deg}$.

sponding to the angle of static equilibrium. Hence, the good overall correspondence between the CFD and experimental results further fortifies the reliability of the laminar Pseudo-3D method.

\subsection{Buffeting analysis}

Finally, we test the applicability of the introduced turbulent Pseudo-3D VPM by conducting a buffeting analysis. The analysis is performed for both CFD and LU models with the wind characteristics noted in Sec. 4.3. The mean wind speed of
$U=30 \mathrm{~m} / \mathrm{s}$ is selected, to be high enough for vortex shedding effects not to influence the response significantly, and low enough to minimize the possible nonlinear effects. Both of these phenomena are not captured by the LU model. The analyses are performed for time amounting to $t=700 \mathrm{~s}$ with approximately 41 thousand time-steps. The fluid domain for each slice is discretized by approximately $N_{p}=175$ thousand particles, resulting in a total of $N_{p}=8.75$ million particles for all slices (cf. Fig. 20). A parallel multi-core CPU architecture is utilized and each slice is run on a single core. The runtime was approximately $100 \mathrm{~h}$, excluding the time required for generation of 


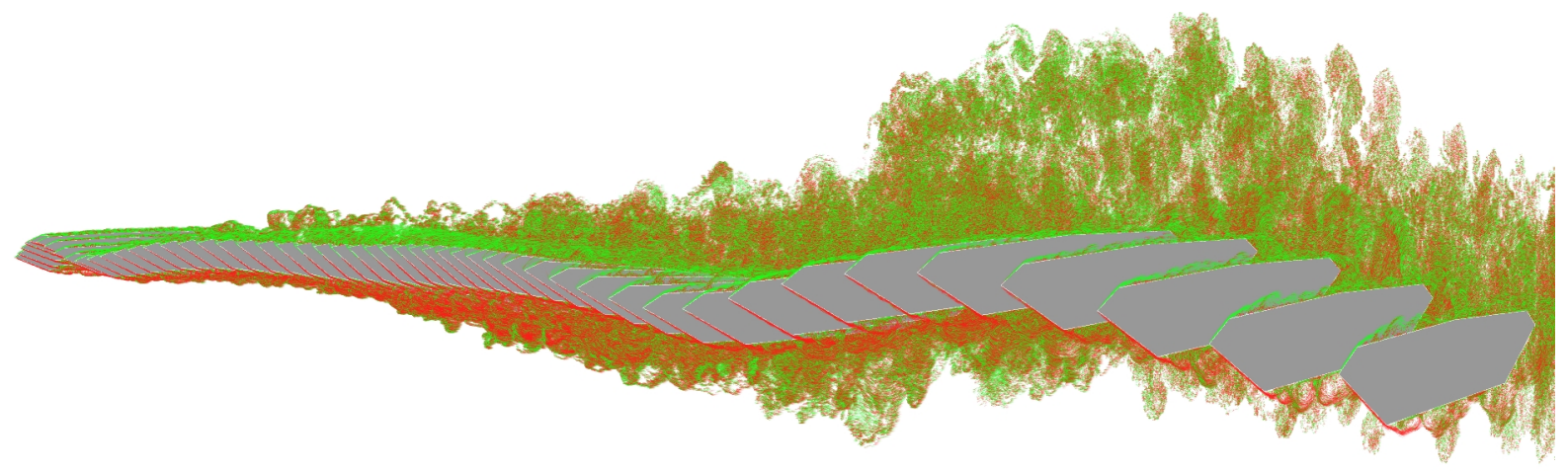

Figure 18: Instantaneous particle map for the Pseudo-3D flutter analysis under laminar flow during LCO at maximum positive rotation (nose-up).

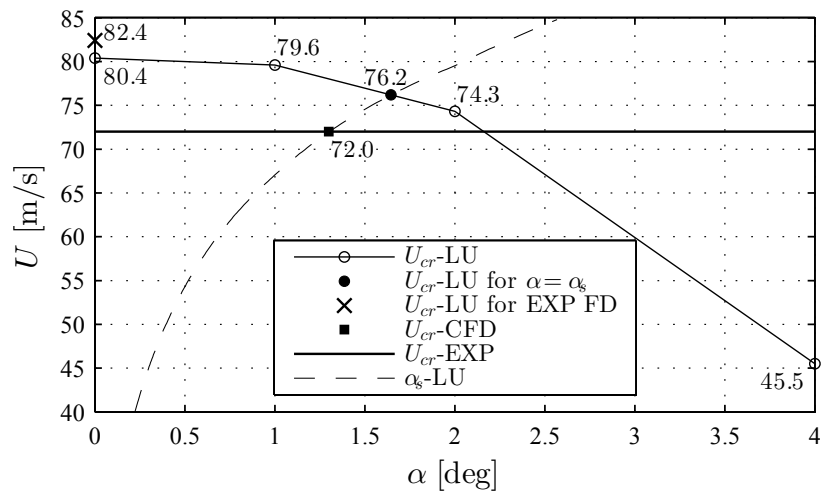

Figure 19: Critical flutter velocity $U_{c r}$ for the LU and CFD models and from experiments (EXP) for taut strip model [51]. In case of the LU model, $U_{c r}$ is determined for a positive angle of incidence and the corresponding value is computed using linear interpolation at the intersection with the angle at static equilibrium $\alpha_{s}\left(U_{c r}\right.$-LU for $\left.\alpha=\alpha_{s}\right)$. Additionally, the critical velocity is given for the LU model for the experimental flutter derivatives [49] at angle $\alpha=0$ ( $U_{c r}$-LU for EXP FD).

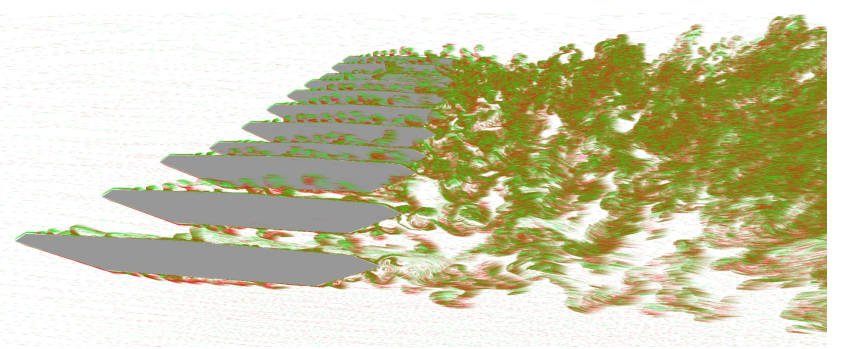

Figure 20: Pseudo-3D buffeting analysis: representative 10 out of 50 slices. The circulation of the inflow particles is low compared to the particles in the wake; thus, their visibility is low.

inflow particles.

Two cases are considered for the free-stream turbulence in the LU model: case one (C1), considering the loss of turbulent energy of the longitudinal fluctuations of the CFD model, that is, $I_{u}=8 \%$ and $I_{w}=6 \%$, and case two (C2), where the turbulence intensity is taken as the prescribed quantities, that is, $I_{u}=11 \%$ and $I_{w}=6 \%$. With this, a comparable basis is maintained between the CFD and LU model for C1, whereas the influence of the loss of lateral turbulent energy on the response can be evaluated by studying $\mathrm{C} 1$ and $\mathrm{C} 2$ for the LU model. For each of the two cases for the LU model, 20 analyses are conducted with randomly generated wind fluctuations in order to facilitate statistical significance of the results. As per common practice for time-domain buffeting analysis with randomly generated wind fluctuations, the $99 \%$ confidence interval of the results is considered for the comparison (cf. e.g. [24]).

A representative sample of the response time histories for the CFD and LU model for C1 are shown in Fig. 21, while Fig. 22 depicts the span-wise root mean square (RMS) of the displacements. In the latter figure, the curve for the LU model in both cases is an average from the 20 analyses, while the shaded area corresponds to the $99 \%$ confidence interval of the response for the $\mathrm{LU}$ model for $\mathrm{C} 1$. The lateral displacements are overestimated from the CFD analysis by $4.4 \%$ compared to the mean value of the response for the $\mathrm{LU}$ model for $\mathrm{C} 1$, which can be attributed to the quasi-steady values used for the lateral aerodynamic damping and the slower decay from the initial impulse in the CFD model. The RMS of the vertical displacements at the span of the CFD model is in a good agreement with the mean value of the $\mathrm{LU}$ for $\mathrm{C} 1$ with a discrepancy of $2 \%$. The difference in the mean value of the RMS of the torsion for the LU model for $\mathrm{C} 1$ is $10 \%$ with respect to the CFD model. Figure 23 depicts the modal contributions of the first three modes for each component of the response. Judging from the relative modal contribution, there is a good correspondence between the CFD and LU model for C1. The PSD of the response at $x / L=0.5$ is shown in Fig. 24, where the gray shaded area corresponds to the minimum and maximum PSD per frequency. Looking at the frequency content of the vertical and rotational displacements, it can be observed that the CFD model results in a slightly increased value in the part of the response due to background turbulence. The shape of the RMS vertical response of CFD model is slightly distorted and unsymmetrical compared to the LU model (cf. Fig. 22). Nevertheless, it is noted that the response for the CFD model is within the confidence interval of the response for the LU model, from which it can 

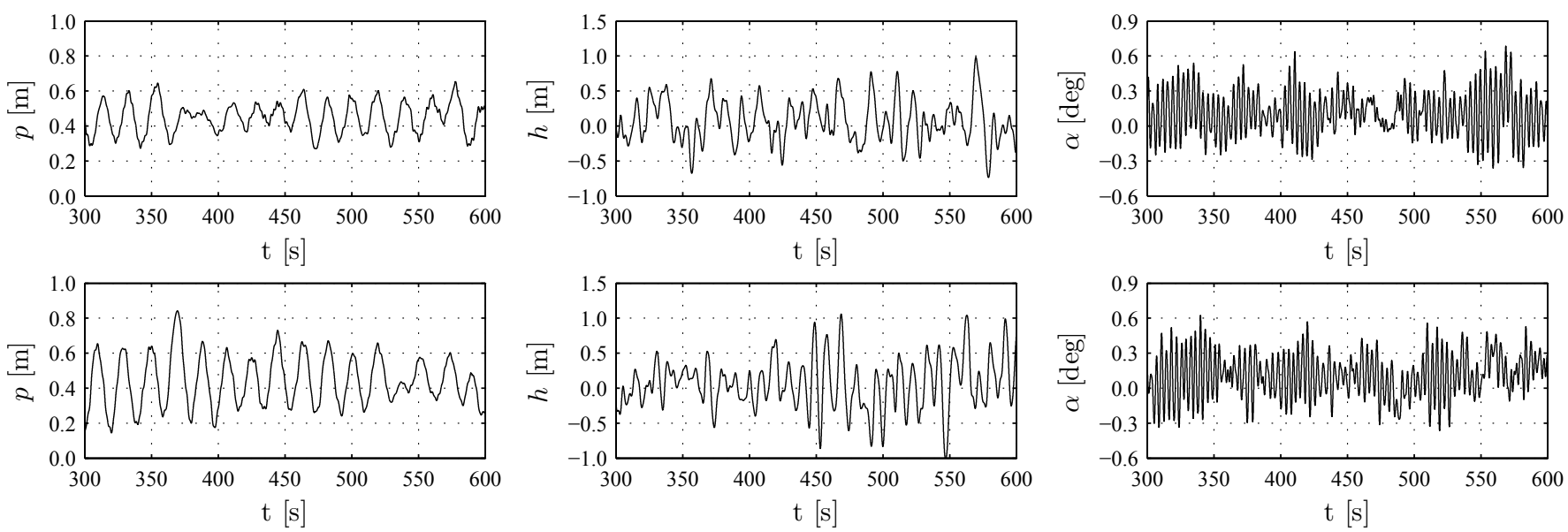

Figure 21: Representative sample time-histories of the lateral (left), vertical (center) and rotational (right) displacements at midspan $(x / L=0.5)$ from the buffeting analysis for the CFD (top) and LU (bottom) model for C1.
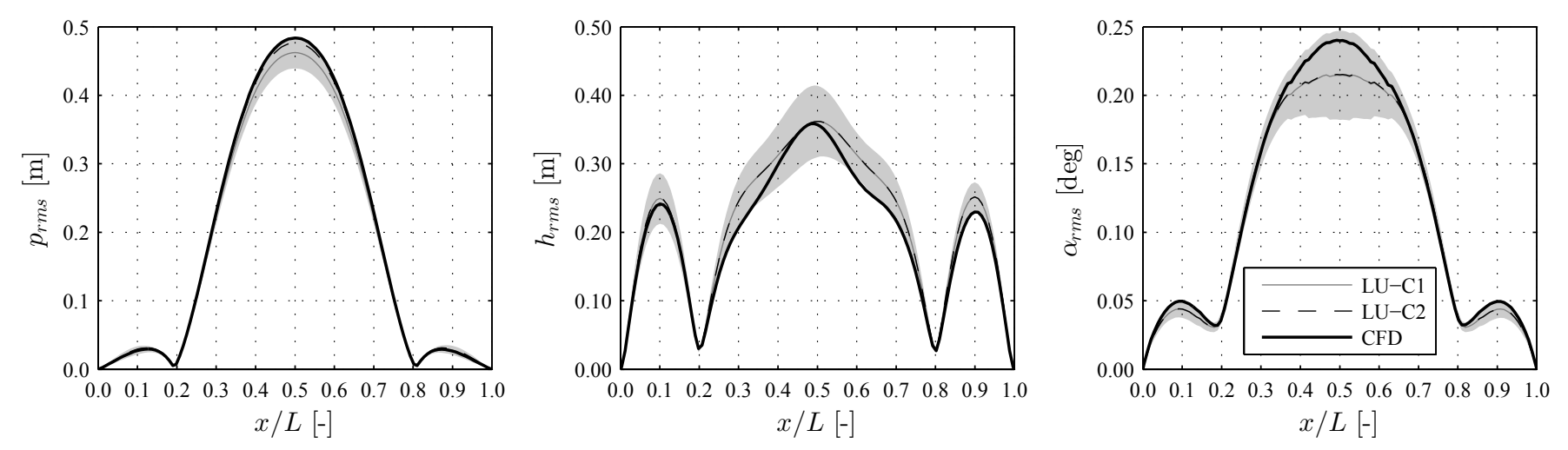

Figure 22: Span-wise RMS of the lateral (left), vertical (center) and rotational (right) displacements for the 3D CFD and LU model. The shape of the response for the LU model in case $\mathrm{C} 1\left(I_{u}=8 \%, I_{w}=6 \%\right)$ and $\mathrm{C} 2\left(I_{u}=11 \%, I_{w}=6 \%\right)$ is obtained as an average from 20 generated random wind time histories with $U=30 \mathrm{~m} / \mathrm{s}$. The shaded area defines the $99 \%$ confidence interval of the displacements for the LU model for $\mathrm{C} 1$.
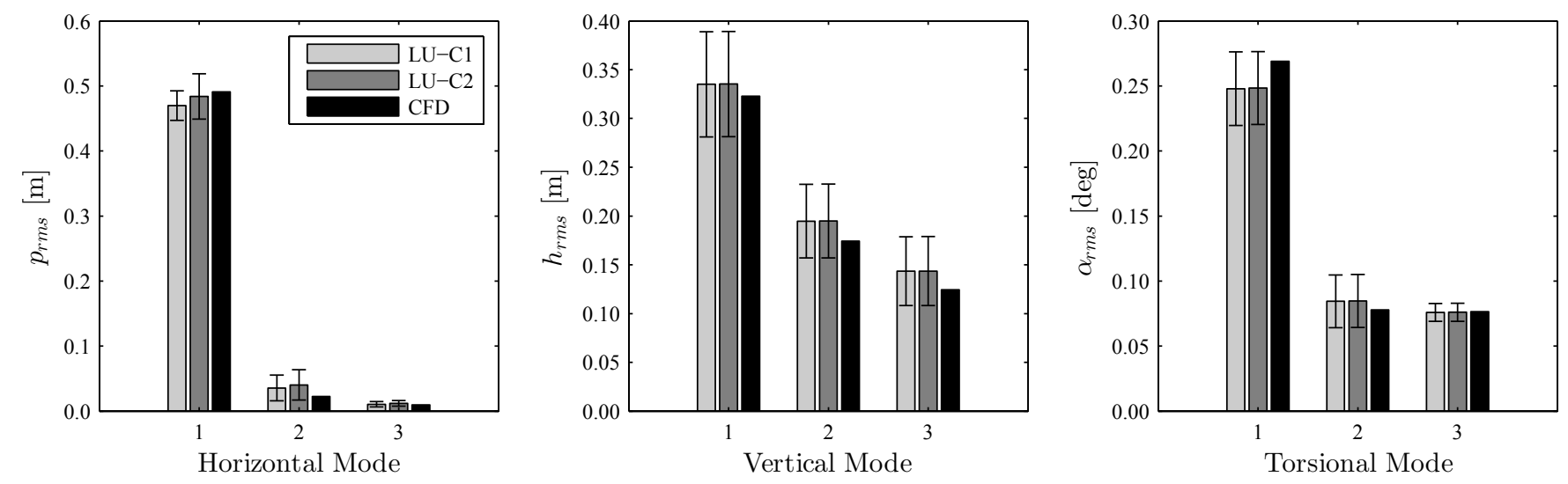

Figure 23: RMS modal contribution of the first three modes of the lateral (left), vertical (center) and rotational (right) displacements for the 3D CFD and LU model. The error bars indicate the 99\% confidence interval of the displacements for the LU model.

be concluded that a good agreement is obtained for the vertical and rotational displacements. The loss of lateral turbulent energy affected only the lateral displacement, where $\mathrm{C} 1$ under- estimated the response compared to the $\mathrm{C} 2$ by $3.1 \%$. Therefore, for bridge decks with small drag coefficient at the angle of static equilibrium compared to the lift slope, which is commonly 

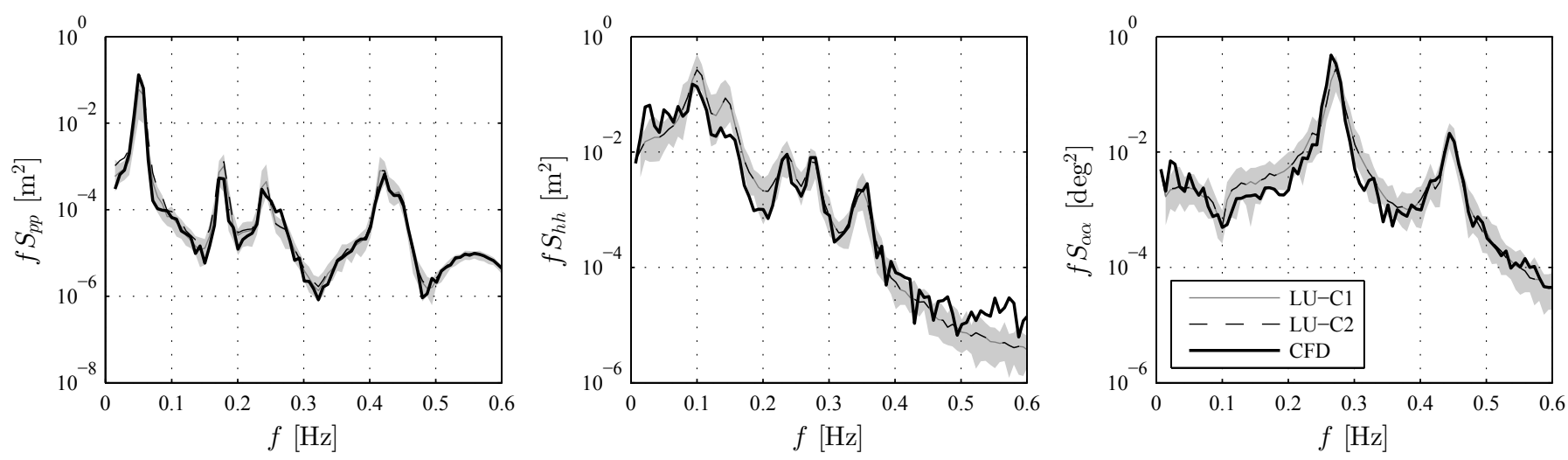

Figure 24: PSD of the of the lateral (left), vertical (center) and rotational (right) displacements at midspan $(x / L=0.5)$ from the buffeting analysis. The shaded area defines the minimum and maximum amplitudes at each frequency component from 20 timehistories for the LU model for $\mathrm{C} 1$.

the case for most of the streamlined sections, the loss of lateral turbulent energy can be negligible for the vertical and torsional displacements.

The full set of experimental aerodynamic coefficients for the LU model, including static wind coefficients, flutter derivatives and aerodynamic admittance, are not available for the section H9.1 of the Great Belt in the literature to the best of authors' knowledge. All these experimental deliverables are necessary for a buffeting analysis using the LU model with experimental aerodynamic coefficients. A validation of a Pseudo-3D method for well-documented aerodynamic coefficients and geometry of a bridge deck in controlled wind tunnel conditions may constitute topic for further study.

\section{Conclusion}

In this study, we extended the Pseudo-3D VPM framework for aeroelastic analyses of long span bridges in terms of methodology, application and comparative assessment.

First, a turbulent Pseudo-3D VPM was introduced by coupling the 2D VTG method for free-stream turbulence with the laminar Pseudo-3D VPM. The span-wise correlation of the wind fluctuations was established based on Taylor's hypothesis. The vertical fluctuations yielded satisfactory results compared to the prescribed turbulence properties, while a loss of correlation and lateral turbulent energy is noted. As shown in an analytical manner, the loss of lateral correlation is a consequence of the cross-PSD of the circulation being mostly based on the PSD of the vertical fluctuations. The partial anisotropy is inherent in the VTG within the wind fluctuation-to-particle conversion procedure. Nevertheless, previously this was not even partially possible in the PTG.

Second, the application of the laminar Pseudo-3D VPM was extended for multimode flutter analysis including LCO in the post-flutter regime. The self-excited forces were taken to be fully correlated between slices. The critical flutter velocity of the laminar Pseudo-3D VPM yielded good agreement with respect to the LU model and experimental results reported in the literature for the Great Belt bridge.
Finally, the turbulent Pseudo-3D VPM was applied for a buffeting analysis of the Great Belt bridge. Good correspondence was noted in the comparison of the displacements for the CFD model and the LU model, despite the discussed deficiencies of the longitudinal fluctuations. The comparison was conducted in a statistical manner. Conducting such comparison, for the first time, demonstrated the reliability of the turbulent Pseudo-3D VPM for aeroelastic analyses with free-stream turbulence.

In conclusion, the laminar and presented turbulent Pseudo3D VPM provide a new framework to unveil some of the nonlinear and local non-stationary effects for wind-bridge interaction. Utilizing the Pseudo-3D VPM, the vortex shedding, local turbulence effects and aerodynamic nonlinearities are inherent in a $2 \mathrm{D}$ manner. Thus, this provides an advantage over the LU model, especially for high amplitudes of oscillation and severe wind conditions. The notion of this study is to show that the Pseudo-3D VPM is readily applicable for aeroelastic analyses of bridges. It is indented that the method is considered from a modeling aspect, rather than as a simulation, serving as a compromise between the limitations of the semi-analytical models and high computational demand of the 3D CFD simulations.

\section{Acknowledgments}

This research is supported by the German Research Foundation (DFG) [Project No. 43475018], which is gratefully acknowledged by the authors.

\section{References}

[1] I. Kavrakov, G. Morgenthal, A comparative assessment of aerodynamic models for buffeting and flutter of long-span bridges, Eng 3 (2017) 823838. doi:10.1016/j.eng.2017.11.008.

[2] T. Wu, A. Kareem, A nonlinear convolution scheme to simulate bridge aerodynamics, Comput Struct 128 (2014) 259-271. doi:j.compstruc.2013.06.004.

[3] G. Diana, D. Rocchi, T. Argentini, S. Muggiasca, Aerodynamic instability of a bridge deck section model: Linear and nonlinear approach to force modeling, J Wind Eng Ind Aerodyn 98 (2010) 363-374. doi:10.1016/j.jweia.2010.01.003. 
[4] G. Diana, D. Rocchi, T. Argentini, An experimental validation of band superposition model of the aerodynamic forces acting on a multibox deck sections, J Wind Eng Ind Aerodyn 113 (2013) 40-58. doi:10.1016/j.jweia.2012.12.005.

[5] T. Wu, A. Kareem, A nonlinear analysis framework for bluffbody aerodynamics: A Volterra representation of the solution of Navier-Stokes equations, J Fluids Struct 54 (2015) 479-502. doi:10.1016/j.jfluidstructs.2014.12.005.

[6] T. Abbas, I. Kavrakov, G. Morgenthal, Methods for flutter stability analysis of long-span bridges: a review, Proc Inst Civ Eng - Bridge Eng 170 (2017) 271-310. doi:10.1680/jbren.15.00039.

[7] J. Walther, A. Larsen, Two dimensional discrete vortex method for application to bluff body aerodynamics, J Wind Eng Ind Aerodyn 67\&68 (1997) 183-193. doi:10.1016/S0167-6105(97)00072-X.

[8] A. Larsen, J. H. Walther, Aeroelastic analysis of bridge girder sections based on discrete vortex simulations, J Wind Eng Ind Aerodyn 67\&68 (1997) 253-265. doi:10.1016/S0167-6105(97)00077-9.

[9] I. J. Taylor, M. Vezza, A numerical investigation into the aerodynamic characteristics and aeroelastic stability of a footbridge, J Fluids Struct 144 (2009) 155-177. doi:10.1016/j.jfluidstructs.2008.05.001.

[10] H. Y. Farsani, D. T. Valentine, A. Arena, W. Lacarbonara, P. Marzocca, Indicial functions in the aeroelasticity of bridge decks, J Bridge Eng 19 (2014) 1-13. doi:10.1016/j.jfluidstructs.2014.02.015

[11] G. Morgenthal, F. McRobie, A comparative study of numerical methods for fluid-structure interaction analysis in long-span bridge design, Wind Struct 5 (2002) 101-114. doi:10.12989/was.2002.5.2_3_4.101.

[12] J. Prendergast, F. McRobie, Simulation of 2D unsteady wind by a vortex method and application to studying bluff body flow, in: 7th UK Conf Wind Eng, 2006.

[13] J. Prendergast, Simulation of 2D unsteady wind by a vortex method, Ph.D. thesis, Cambridge University (2007).

[14] J. T. Rasmussen, M. M. Hejlesen, A. Larsen, J. H. Walther, Discrete vortex method simulations of the aerodynamic admittance in bridge aerodynamics, J Wind Eng Ind Aerodyn 99 (2010) 776-785. doi:10.1016/j.jweia.2010.06.011.

[15] M. M. Hejlesen, J. T. Rasmussen, A. Larsen, J. H. Walther, On estimating aerodynamic admittance of bridge section by mesh-free vortex method, J Wind Eng Ind Aerodyn 146 (2015) 117-127. doi:10.1016/j.jweia.2015.08.003.

[16] I. Kavrakov, G. Morgenthal, A synergistic study of a CFD and semi-analytical models for aeroelastic analysis of bridges in turbulent wind conditions, J Fluid Struct 82 (2018) 59-85. doi:10.1016/j.jfluidstructs.2018.06.013.

[17] A. Belver, A. Ibán, R. Rossi, Lock-in and drag amplification effects in slender line-like structures trough CFD, Wind Struct 15 (3) (2012) 189 208. doi:10.12989/was.2012.15.3.189.

[18] G. Morgenthal, A. Corriols, B. Bendig, A GPU-accelerated Pseudo-3D vortex method for aerodynamic analysis, J Wind Eng Ind Aerodyn 125 (2014) 69-80. doi:10.1016/j.jweia.2013.12.002.

[19] T. Abbas, Assessment of numerical prediction models for aeroelastic instabilities of bridges, Ph.D. thesis, Bauhaus-University Weimar (2016).

[20] K. I. Tolba, G. Morgenthal, Pseudo three-dimensional simulation of aeroelastic response to turbulent wind using vortex particle methods, J Fluids Struct 72 (2017) 1-24. doi:10.1016/j.jfluidstructs.2017.04.001

[21] A. G. Davenport, The response of slender, line-like structures to a gusty wind, Proc Inst Civ Eng 23 (1962) 389-408. doi:10.1680/iicep.1962.10876.

[22] R. H. Scanlan, The action of flexible bridges under wind, I: Flutter theory, J Sound Vib 60 (2) (1978) 187-199. doi:10.1016/S0022-460X(78)800285.

[23] R. H. Scanlan, The action of flexible bridges under wind, II: Buffeting theory, J Sound Vib 60 (2) (1978) 201-211. doi:10.1016/S0022460X(78)80029-7.

[24] X. Chen, M. Matsumoto, A. Kareem, Time domain flutter and buffeting response analysis of bridges, J Eng Mech 126 (1) (2000) 7-16 doi:10.1061/(ASCE)0733-9399(2000)126:1(7).

[25] A. Jain, N. P. Jones, R. H. Scanlan, Coupled flutter and buffeting analysis of long-span bridges, J Struct Eng 122 (7) (1996) 716-725. doi:10.1061/(ASCE)0733-9445(1996)122:7(716).

[26] R. H. Scanlan, J. G. Béliveau, K. S. Budlong, Indicial aerodynamic functions for bridge decks, J Eng Mech 100 (1974) 657-672.
[27] M. Lazzari, R. V. Vitalini, A. V. Saetta, Aeroelastic forces and dynamic response of long-span bridges, Int J Num Meth Eng 60 (2004) 10111048. doi:10.1002/nme.987.

[28] L. Caracoglia, N. P. Jones, Time domain vs frequency domain characterization of aeroelastic forces for bridge deck sections, J Wind Eng Ind Aerodyn 91 (2003) 371-402. doi:10.1016/S0167-6105(02)00399-9.

[29] G. Cottet, P. Koumoutsakos, Vortex methods: Theory and Practice, Cambridge University Press, 2000

[30] G. Morgenthal, J. Walther, An immersed interface method for the Vortex-In-Cell algorithm, Comput Struct 85 (2007) 712-726. doi:j.compstruc.2007.01.020.

[31] A. Leonard, Vortex methods for flow simulation, J Compt Phys 37 (1980) 2891-355, doi:10.1016/0021-9991(80)90040-6.

[32] A. Chorin, Numerical study of slightly viscous flow, J Fluid Mech 27 (1973) 785-796. doi:10.1017/S0022112073002016.

[33] R. Hockney, J. Eastwood, Computer Simulation Using Particles, Taylor \& Francis, 1988.

[34] M. Shinozuka, G. Deodatis, Simulation of multi-dimensional Gaussian stochastic fields by spectral representation, Appl Mech Rev 49 (1996) 29-53. doi:10.1115/1.3101883.

[35] G. Solari, G. Piccardo, Probabilistic 3-D turbulence modeling for gust buffeting of structures, Prob Eng Mech 16 (2001) 73-86. doi:10.1016/S0266-8920(00)00010-2.

[36] Q. Ding, L. Zhu, H. Xiang, An efficient ergodic simulation of multivariate stochastic process with spectral representation, Prob Eng Mech 26 (2011) 350-356. doi:10.1016/j.probengmech.2010.09.006.

[37] S. Chawdhury, G. Morgenthal, Flow reproduction using vortex particle methods for simulating wake buffeting response of bluff structures, J Wind Eng Ind Aerodyn 151 (2016) 122-136. doi:10.1016/j.jweia.2016.02.003.

[38] R. H. Scanlan, Amplitude and turbulence effects on bridge flutter derivatives, J Struct Eng 123 (2) (1997) 232-236. doi:10.1061/(ASCE)07339445(1997)123:2(232).

[39] Y. Bai, D. Sun, J. Lin, Three dimensional numerical simulations of longspan bridge aerodynamics, using block-iterative coupling and DES, Comput Fluids 39 (2010) 1549-1561. doi:10.2516/ogst:2002004.

[40] Y. Ge, H. Tanaka, Aerodynamic flutter analysis of cable-supported bridges by multi-mode and full-mode approaches, J Wind Eng Ind Aerodyn 86 (2000) 123-153. doi:10.1016/S0167-6105(00)00007-6.

[41] L. Kristensen, On longitudinal spectral coherence, Boundary-Layer Meteorol 16 (1979) 145-153. doi:10.1007/BF02350508.

[42] L. Larose, The dynamic action of gusty winds on long-span bridges, Tech. Rep. BYG-Rapport R-029, Technical University of Denmark (2002).

[43] M. Massaro, J. Graham, The effect of three-dimensionality on the aerodynamic admittance of thin sections in free stream turbulence, J Fluids Struct 57 (2015) 81-90. doi:10.1016/j.jfluidstructs.2015.05.012.

[44] G. Diana, F. Resta, D. Rocchi, A new numerical approach to reproduce bridge aerodynamic non-linearities in the time domain, J Wind Eng Ind Aerodyn 96 (2008) 1871-1884. doi:10.1016/j.jweia.2008.02.052.

[45] J. Mann, The spatial structure of neutral atmospheric surface-layer turbulence, J Fluid Mech 273 (1994) 141-168. doi:10.1017/S0022112094001886

[46] F. Tubino, F. Solari, Gust buffeting of long span bridges: Double modal transformation and effective turbulence, Eng Struct 29 (2007) 1698-1707. doi:10.1016/j.engstruct.2006.09.019.

[47] X. Chen, M. Matsumoto, A. Kareem, Aerodynamic coupling effects on flutter and buffeting of bridges, J Eng Mech 126 (1) (2000) 17-26. doi:10.1061/(ASCE)0733-9399(2000)126:1(17).

[48] O. Øiseth, A. Rönnquist, Sigbjörnsson, Effects of co-spectral densities of atmospheric turbulence on the dynamic response of cable-supported bridges: A case study, J Wind Eng Ind Aerodyn 116 (2013) 83-93. doi:10.1016/j.jweia.2013.03.001.

[49] T. Reinhold, M. Brinch, A. Damsgaard, Wind tunnel tests for the Great Belt Link, in: A. Larsen (Ed.), Aerodynamics of Large Bridges, Copenhagen, Denmark, 1992, pp. 255-267.

[50] G. Morgenthal, Advances in numerical bridge aerodynamic$s$ and recent applications, Struct Eng Int 2 (2005) 95-100. doi:10.2749/101686605777963161.

[51] A. Larsen, Aerodynamic aspects of the final design of the $1624 \mathrm{~m}$ suspension bridge across the Great Belt, J Wind Eng Ind Aerodyn 48 (1993) 261-285. doi:10.1016/0167-6105(93)90141-A. 
[52] R. H. Scanlan, Motion-related body-force functions in twodimensional low-speed flow, J Fluids Struct 14 (2000) 49-63. doi:10.1006/jfls.1999.0252.

[53] G. L. Larose, Experimental determination of the aerodynamic admittance of a bridge deck segment, J Fluids Struct 13 (1999) 1029-1040. doi:10.1006/jfls.1999.0244.

[54] M. Gu, X.-R. Qin, Direct identification of flutter derivatives and aerodynamic admittances of bridge decks, Eng Struct 26 (2004) 2161-2172. doi:10.1016/j.engstruct.2004.07.015.

[55] C. B. Kim, S. S. Yhim, Buffeting analysis of cable-stayed bridge using three-dimensional computational fluid dynamics, J Bridge Eng 19 (2014) 319-329. doi:10.1061/(ASCE)BE.1943-5592.0000618.

[56] Y. Ge, S. Liu, K. Xu, K. Li, J. Xia, 2D CFD simulation of wind-induced responses of bridge decks based on fluid-structure interaction, in: BBAA 8, Boston, USA, 2016.

[57] S. Chawdhury, G. Morgenthal, Numerical simulations of aeroelastic instabilities to optimize the performance of flutter-based electromagnetic energy harvesters, J Intell Material Syst 29 479-495. doi: $10.1177 / 1045389 X 17711784$.

[58] M. Matsumoto, Y. Daito, F. Yoshizumi, Y. Ichikawa, T. Yabutani, Torsional flutter of bluff bodies, J Wind Eng Ind Aerodyn 93 (1997) 69-71. doi:10.1016/S0167-6105(97)00213-4.

[59] X. Chen, A. Kareem, Nonlinear response analysis of long-span bridges under turbulent winds, J Wind Eng Ind Aerodyn 89 (2001) 1335-1350. doi:10.1016/S0167-6105(01)00147-7. 